\title{
Review
}

Jie Zhou, Jiajie Chen, Yanqi Ge and Yonghong Shao*

\section{Two-dimensional nanomaterials for Förster resonance energy transfer-based sensing applications}

https://doi.org/10.1515/nanoph-2020-0065

Received January 30, 2020; revised March 6, 2020; accepted March 7, 2020

\begin{abstract}
Förster resonance energy transfer (FRET)-based sensing has been steadily gaining popularity in the areas of biochemical analysis, environmental monitoring, and disease diagnosis in the past 20 years. Two-dimensional (2D) nanomaterials are extensively used as donors and acceptors in the FRET sensing because of their attractive optical and chemical properties. In this review, we first present the FRET theory and calculations to give readers a better understanding of the FRET phenomenon. Then, we discuss the recent research advances in using 2D nanomaterials as donors and acceptor in FRET sensing. Finally, we summarize the existing challenges and future directions of 2D nanomaterials in the FRET sensing applications.
\end{abstract}

Keywords: 2D nanomaterials; FRET sensing; donor; acceptor; 2D quantum dots.

\footnotetext{
*Corresponding author: Yonghong Shao, College of Physics and Optoelectronic Engineering, Key Laboratory of Optoelectronic Devices and Systems of Ministry of Education and Guangdong Province, Shenzhen University, Shenzhen 518060, People's Republic of China, e-mail: shaoyh@szu.edu.cn. https://orcid. org/0000-0001-6373-8545

Jie Zhou and Jiajie Chen: College of Physics and Optoelectronic Engineering, Key Laboratory of Optoelectronic Devices and Systems of Ministry of Education and Guangdong Province, Shenzhen University, Shenzhen 518060, People's Republic of China Yanqi Ge: Institute of Microscale Optoelectronics, Collaborative Innovation Centre for Optoelectronic Science and Technology, Key Laboratory of Optoelectronic Devices and Systems of Ministry of Education and Guangdong Province, College of Physics and Optoelectronic Engineering, Shenzhen Key Laboratory of MicroNano Photonic Information Technology, Guangdong Laboratory of Artificial Intelligence and Digital Economy (SZ), Shenzhen University, Shenzhen 518060, People's Republic of China
}

\section{Introduction}

The Förster resonance energy transfer (FRET) theory, first discovered by J. Perrin in the early 20th century and later founded by Förster in the 1950s [1], has been widely used in the spectral analysis for a variety of fluorescent applications. The FRET process would occur when a donor in the excited state (normally a fluorophore) transfers energy to a proximal acceptor in the ground state through a longdistance (10-100 ̊) dipole-dipole interaction, which is a nonradiative process [2]. Consequently, the emission of donors would be quenched by the acceptors. The FRET phenomenon is extremely sensitive to the distance between the donor and the acceptor, exhibiting great capability in the sensing of molecular interactions [3]. Besides the distance, the rate and efficiency of the energy transfer are dependent on several other factors, such as the quantum yield of the donor and the quenching efficiency of the receptor. Thus, the optical properties of the FRET pairs are of great importance to the performance of FRET sensors.

The development of nanomaterials would be beneficial for the fabrication of FRET sensors to achieve better performance. Compared with traditional dyes and fluorescent proteins, the fluorescent nanomaterials, for instance, upconversion nanoparticles (UCNPs) [4, 5], semiconductor quantum dots (QDs) [6, 7], metal nanoclusters (NCs) [8], polymer dots [9, 10], and carbon dots [11, 12], have higher quantum yield, stronger photostability, and longer fluorescence lifetime, so they have been extensively utilized as donors in the FRET sensors. Moreover, some nanomaterials, for instance, gold nanoparticles (AuNPs) $[13,14]$, gold nanorods [15], and carbon nanotubes (CNTs) $[16,17]$, have been widely employed as efficient fluorescence quenchers in the FRET sensors owing to their excellent optical quenching capabilities.

Recently, two-dimensional (2D) nanomaterials have been steadily gaining popularity in the fields of FRET sensing because of their excellent optical and chemical properties. Compared with other nanomaterials being used 
in the FRET sensors, 2D nanomaterials have some inherent advantages such as high chemical and photostability, large specific surface area, high adsorption ability toward chemicals or biomolecules, good biocompatibility, low toxicity, layer- and size-dependent optical property, and so on [18-25]. The different luminescent and optical absorption properties of various $2 \mathrm{D}$ nanomaterials enable them to act as either donors or acceptors in the FRET sensors. Some 2D nanomaterials, for instance, graphitic-phase $\mathrm{C}_{3} \mathrm{~N}_{4}\left(\mathrm{~g}-\mathrm{C}_{3} \mathrm{~N}_{4}\right)$ [26], metal-organic frameworks (MOFs) [27], selenium [28], perovskite [29], and 2D nanomaterial-derived QDs (2D-QDs) [30], could emit strong photoluminescence (PL) and have been frequently employed as donors in the FRET sensing. On the other hand, the $2 \mathrm{D}$ nanomaterials with zero or small bandgap could be utilized as acceptors or quenchers in the FRET sensors. It was found that the acceptor could directly quench the fluorescence of the donor without reradiating the transferred energy via a FRET mechanism if the bandgap of the acceptor is small enough [31]. The 2D nanomaterials exhibit better quenching capability than normal nanoquenchers. Taking graphene as an example, it was first found to be a super quencher that could quench the fluorescence of donor molecule at a distance up to approximately $300 \AA$ A demonstrating its long-range energy transfer properties [32, 33]. In addition, the graphene exhibits a broad absorption band from the UV region to the nearinfrared (NIR) region because of its heterostructure, for which it could act as a broad-spectrum quencher for the fluorescent donor. Other 2D nanomaterials, such as transition metal dichalcogenides (TMDs) [34]; black phosphorene (BP) $[35,36]$; transition metal carbides, nitrides, and carbonitrides (MXenes) [37]; and $\mathrm{MnO}_{2}$ [38], have also been successfully employed as acceptors in the FRET sensors.

Many review articles about GQDs and 2D graphenebased FRET sensing have already been presented [39, 40]. In this article, we mainly review the recent applications of 2D nanomaterials except graphene in FRET sensing. The article is categorized in five sections as below. After the Introduction part, in Section 2, we expound the FRET theory and related calculations. In Sections 3 and 4, we present the state of the art of applications of 2D nanomaterials as energy donors and acceptors for FRET sensing. Finally, in Section 5, we conclude the challenges and future directions of 2D nanomaterial-based FRET sensors.

\section{FRET theory and calculations}

During the FRET process, the energy donor $(D)$ obtains energy from the excitation source and transfers the energy in a nonradioactive manner to the nearby energy acceptor
(A). The acceptor could either re-radiate the adsorbed energy or not. This process can be represented as follows:

$$
\begin{gathered}
D+h v \rightarrow D^{*} \\
D^{*}+A \rightarrow D+A^{*} \\
A^{*} \rightarrow A+h v
\end{gathered}
$$

where $h$ is the Planck's constant, and $v$ is the frequency of the radiation.

The energy transfer is characterized by the fluorescence quenching of the donor and the decreased lifetime of the excited state, along with the increased fluorescence intensity of the acceptor if the acceptor has the PL capacity. In the presence of the acceptor, the donor would directly transfer its excited energy to the acceptor rather than emitting photons.

The occurrence of the FRET must meet several criteria: (i) The absorption spectrum of the acceptor should overlap with the fluorescence emission spectrum of the donor. (ii) The donor should have an appropriate distance (typically 10-100 Å) to the receptor. (iii) The transition dipole orientation of the acceptor should be approximately parallel to that of the donor. (iv) The fluorescence lifetime of the donor should be long enough for generating the FRET.

J. Perrin found the FRET phenomenon in the fluorescence-quenching experiment and put forward that two molecules that do not collide can interact with each other within a distance greater than the molecular diameter through the dipole-dipole interaction. About 20 years later, Förster proposed an improved theory based on the discovery of Perrin [41], which provided a quantitative interpretation of nonradiative energy transfer as expressed by (4):

$$
k_{T}(r)=\frac{1}{\tau_{D}}\left(\frac{R_{0}}{r_{\mathrm{DA}}}\right)^{6}
$$

where $k_{T}(r)$ is the rate of energy transfer from the donor to the acceptor, $\tau_{D}$ is the lifetime of the donor in the absence of the acceptor, $r_{\mathrm{DA}}$ is the distance between the donor and the acceptor, and $R_{0}$ is the Förster radius referring to the distance when the efficiency of energy transfer is 50\% defined by (5) and (6):

$$
\begin{gathered}
R_{0}^{6}=\left[\frac{9000(\ln 10) k^{2} \varnothing_{d}}{128 \pi^{5} N n^{4}}\right] J(\lambda) \\
J(\lambda)=\int_{0}^{\alpha} F_{D}(\lambda) \varepsilon_{A}(\lambda) \lambda^{4} d \lambda
\end{gathered}
$$


where $J(\lambda)$ is the spectral overlap integral of the absorption spectrum of the acceptor and the emission spectrum of the donor, $\varnothing_{d}$ is the fluorescence quantum yield of the donor in the absence of the acceptor, $n$ is the refractive index of the medium, $k^{2}$ is the orientation factor of transition dipole moments between the donor and the acceptor, and $N$ is the Avogadro coefficient. $J(\lambda)$ is determined by the value of $F_{D}(\lambda)$ (the normalized fluorescence intensity of the donor in the wavelength range from $\lambda$ to $d(\lambda)$ and $\varepsilon_{A}(\lambda)\left(\mathrm{M}^{-1} \mathrm{~cm}^{-1}\right.$, the extinction coefficient of the acceptor in the wavelength range from $\lambda$ to $d \lambda$ ).

Equation (1) can also be abbreviated to (7), and the unit of $R_{0}$ is angstrom:

$$
R_{0}=0.2108\left\{k^{2} n^{-4} \Phi_{D} J(\lambda)\right\}^{1 / 6}
$$

The efficiency of energy transfer (E) can be expressed as (8):

$$
E=\frac{k_{T}(r)}{k_{T}(r)+\tau_{D}^{-1}}=\frac{\tau_{D} k_{T}(r)}{1+\tau_{D} k_{T}}
$$

Combining (1) and (5), $E$ can be expressed as (9):

$$
E=\frac{R_{0}^{6}}{R_{0}^{6}+r^{6}}
$$

$E$ can also be expressed as (10):

$$
E=1-\frac{F_{\mathrm{DA}}}{F_{D}}
$$

where $F_{\mathrm{DA}}$ refers to the relative fluorescence intensity of the donor in the presence of the acceptor, and $F_{\mathrm{D}}$ refers to the fluorescence intensity of the donor in the absence of the acceptor.

Finally, the donor-acceptor distance $\left(r_{\mathrm{DA}}\right)$ can be calculated by (11), in which $r_{\mathrm{DA}}$ should be in the range of 10-100 Å to achieve high FRET efficiency:

$$
r_{\mathrm{DA}}{ }^{6}=\frac{R_{0}{ }^{6}(1-E)}{E}
$$

\section{2D nanomaterials as the donor in FRET sensors}

\subsection{D-QDs as the donor in FRET sensors}

When the 2D nanomaterials are reduced to OD nanomaterials with lateral sizes smaller than $20 \mathrm{~nm}$, that is, $2 \mathrm{D}-\mathrm{QDs}$, it would start to emit fluorescence due to the quantum confinement effects. Besides graphene QDs, TMD QDs have also been well explored. In contrast to the zero-gap graphene, the semiconductive TMDs possess narrow and tunable bandgap that is appreciated in optoelectronic and electronic applications [42-45]. The general chemical formula of TMDs is $M X_{2}$, in which $M$ represents a transition metal atom, and $X$ denotes a chalcogen atom. The single-layered TMDs are formed with one intermediate sublayer of transition metals sandwiched by two sublayers of chalcogen atoms. The atoms are arranged in a hexagonal shape in one plane. Two-dimensional $\mathrm{MoS}_{2}$ is the most widely studied 2D nanomaterial in the TMD family because of its excellent physical properties and the availability of bulk $\mathrm{MoS}_{2}$ in nature [46]. $\mathrm{MoS}_{2}$ QDs, one form of $2 \mathrm{D} \mathrm{MoS}_{2}$ with a lateral size smaller than $10 \mathrm{~nm}$, show superior optical properties because of the boundary effects and quantum confinement effects. Moreover, $\mathrm{MoS}_{2} \mathrm{QDs}$ exhibit good chemical stability, low toxicity, and superior biocompatibility. Therefore, $\mathrm{MoS}_{2}$ QDs are considered to be an ideal material for fluorescence detection. Table 1 lists recent studies relevant to the $2 \mathrm{D}$ nanomaterials except graphene as the donor in FRET sensing.

Ha et al. [63], for the first time, utilized the $\mathrm{MoS}_{2}$ QDs as a fluorescent tag in a FRET system. As shown in Figure 1, the monolayer $\mathrm{MoS}_{2}$ QDs were synthesized by the Li intercalation method, and they emitted a blue luminescence at $415 \mathrm{~nm}$ under the UV excitation. The product generated by the hybridization of ployG-labeled ssDNA and Alexa Fluor $430\left(\mathrm{AF}_{430}\right)$-labeled ssDNA was adsorbed on the $\mathrm{MoS}_{2}$ QDs through the van der Waals (VdW) force between ployG and $\mathrm{MoS}_{2}$. As a result, the distance of $\mathrm{AF}_{430}$ and $\mathrm{MoS}_{2}$ depended on the number of base pairs, which further affected the FRET efficiency between the $\mathrm{MoS}_{2}$ (donor) and $\mathrm{AF}_{430}$ (acceptor) upon the irradiation of $300-\mathrm{nm}$ UV light. At the optimal number of base pairs (13) that corresponded to $4.42 \mathrm{~nm}$ of donor-acceptor distance, the FRET efficiency reached a maximum of $11.73 \%$. In a shorter distance, the quenching mechanism would dominate rather than the FRET. Also, the energy transfers between the $\mathrm{MoS}_{2}$ and $\mathrm{AF}_{430}$ would turn inefficient in a longer distance. In another circumstance, when the conjugates were excited by a light source with a wavelength of $430 \mathrm{~nm}$, the emission of $\mathrm{AF}_{430}$ at $530 \mathrm{~nm}$ would be quenched by the $\mathrm{MoS}_{2}$ as the acceptor. The quenching efficiency was also determined by the number of base pairs, and the quenching effect became insignificant when the number was greater than 13. The results of this study indicated that the role of 2D nanomaterials in the FRET system depended on the several important factors such as the wavelength of the 
Table 1: Summary of the FRET sensing applications of 2D nanomaterials as donors.

\begin{tabular}{|c|c|c|c|c|}
\hline Target analyst & Donor: acceptor pairs & Dynamic range & Detection limit & Reference \\
\hline Bilirubin & $\mathrm{MoS}_{2}$ QDs: bilirubin & $0.5-10.0 \mu \mathrm{m}$ & $2.1 \mathrm{~nm}$ & [47] \\
\hline MicroRNA & $\mathrm{MoS}_{2}$ QDs: FAM-MBs & $5-150 \mathrm{~nm}$ & $0.38 \mathrm{~nm}$ & [48] \\
\hline EP & $\mathrm{MoS}_{2}$ QDs: $P_{\text {EP.PEI }}$ copolymers & $0.2-40 \mu \mathrm{m}$ & $0.05 \mu \mathrm{M}$ & [49] \\
\hline AA & $\mathrm{MoS}_{2}$ QDs: $P_{\text {EP-PEI }}$ copolymers & $0.5-40 \mu \mathrm{M}$ & $0.2 \mu \mathrm{m}$ & [49] \\
\hline 6-MP & $\mathrm{MoS}_{2}$ QDs: DAP & $0.5-70 \mu \mathrm{M}$ & $0.29 \mu \mathrm{M}$ & [50] \\
\hline BSA & $\mathrm{MoS}_{2} \mathrm{QDS}: \mathrm{RGO}$ & $5-50 \mathrm{~nm}$ & NM & [51] \\
\hline Dopamine & $\begin{array}{l}\operatorname{MoS}_{2} \text { QDs-aptamer: } \mathrm{MoS}_{2} \\
\text { nanosheets }\end{array}$ & $0.1-1000 \mathrm{~nm}$ & $45 \mathrm{pm}$ & [52] \\
\hline BSA & $\mathrm{MoS}_{2}$ QDs: polyaniline & $10-70 \mathrm{~nm}$ & $9.86 \mathrm{~nm}$ & [53] \\
\hline GSH & $\mathrm{MoS}_{2}$ QDs: R6G & $5-50 \mathrm{~nm}$ & $2.7 \mathrm{~nm}$ & [54] \\
\hline Nitrite & $\mathrm{MoS}_{2}$ QDs: BSA-AuNCs & $0.5-20 \mathrm{mg} / \mathrm{l}$ & $0.67 \mathrm{~nm}$ & [55] \\
\hline NFZ & WS $_{2}$ QDs: NFZ & $0.17-166 \mu \mathrm{M}$ & $0.055 \mu \mathrm{M}$ & [56] \\
\hline DNA & BP QDs: Dabcyl-L probe & $4-4000 \mathrm{pm}$ & $5.9 \mathrm{pm}$ & [57] \\
\hline GSH & $\mathrm{g}-\mathrm{C}_{3} \mathrm{~N}_{4}: \mathrm{MnO}_{2}$ & NM & $0.2 \mu \mathrm{m}$ & [26] \\
\hline $\mathrm{H}_{2} \mathrm{O}_{2}$ & $\mathrm{~g}-\mathrm{C}_{3} \mathrm{~N}_{4}: \mathrm{MnO}_{2}$ & $0-130 \mu \mathrm{M}$ & $1.5 \mu \mathrm{M}$ & [58] \\
\hline Glucose & $\mathrm{g}-\mathrm{C}_{3} \mathrm{~N}_{4}: \mathrm{MnO}_{2}$ & $0-150 \mu \mathrm{M}$ & $1.5 \mu \mathrm{M}$ & [58] \\
\hline Ricin & $\mathrm{g}-\mathrm{C}_{3} \mathrm{~N}_{4}: \mathrm{MnO}_{2}$ & $0.25-50 \mu \mathrm{g} / \mathrm{ml}$ & $190 \mathrm{ng} / \mathrm{ml}$ & [59] \\
\hline Riboflavi & $\mathrm{g}-\mathrm{C}_{3} \mathrm{~N}_{4}$ : riboflavi & $0.4-10 \mu \mathrm{m}$ & $170 \mathrm{~nm}$ & {$[60]$} \\
\hline Metronidazole & $\mathrm{g}-\mathrm{C}_{3} \mathrm{~N}_{4}$ : metronidazole & $0.01-0.10 \mu \mathrm{g} / \mathrm{ml}$ & $0.008 \mu \mathrm{g} / \mathrm{ml}$ & [61] \\
\hline Dopamine & BSA-Au NCs/g- $\mathrm{C}_{3} \mathrm{~N}_{4}$ : dopamine & $0.05-8.0 \mu \mathrm{M}$ & $0.018 \mu \mathrm{M}$ & [62] \\
\hline $\mathrm{Hg}(\mathrm{II})$ & Perovskite: RBED & $20-90 \mu \mathrm{M}$ & $2.36 \mu \mathrm{M}$ & [29] \\
\hline
\end{tabular}

Note: NM means "not mentioned"; the definitions of abbreviations are listed in the abbreviations.
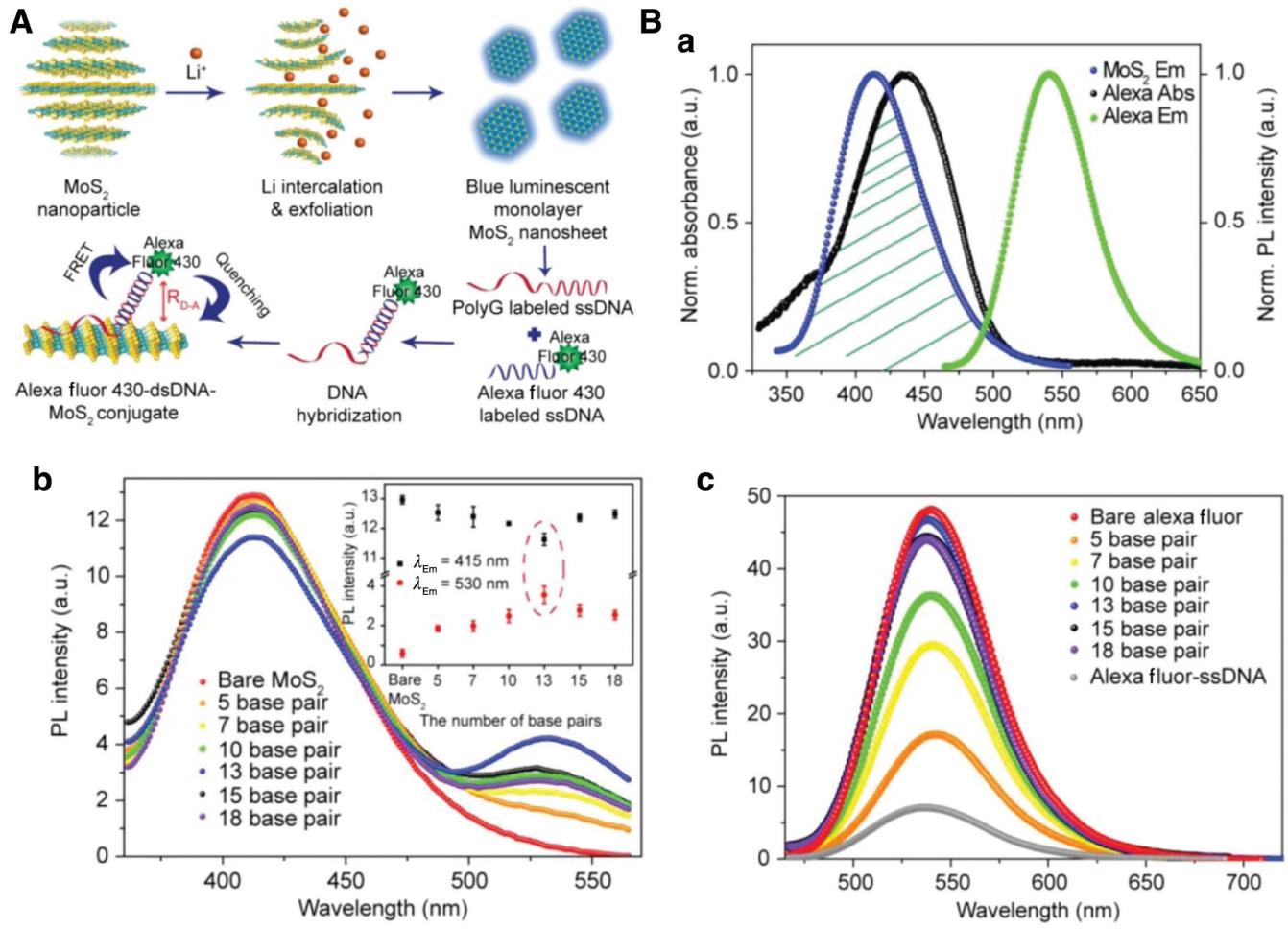

Figure 1: Schematic illustration and experimental results of the $\mathrm{MoS}_{2}$ QDs-based FRET sensor.

(A) Schematic illustration of the $\mathrm{MOS}_{2}$ QDs-based FRET sensor. (B) (a) Normalized spectra of the emission of the MoS ${ }_{2}$ QDs (excitation:

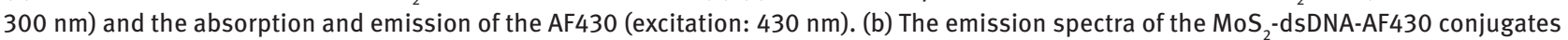
with increasing base pairs of dsDNA (excitation: $300 \mathrm{~nm}$ ). (c) The emission spectra of the AF430 in the MoS $_{2}$-dsDNA-AF430 conjugates with increasing base pairs of dsDNA (excitation: $430 \mathrm{~nm}$ ). Reprinted with permission from Ha et al. [63]. Copyright (2014) John Wiley \& Sons. 
excitation source, the absorption spectrum of the FRET pairs, and the emission spectrum of the FRET pairs.

Since then, the $\mathrm{MoS}_{2}$ QDs have been extensively employed as the donor in the fabrication of FRET sensors. The detection targets acted as either the energy acceptors [47] or the substance that would affect the FRET process [31-38]. Shanmugaraj and John [47] have reported that the bilirubin, as a vital biomarker for hepatic diseases, could directly quench the fluorescence of $\mathrm{MoS}_{2}$ QDs through the FRET process as the absorption of bilirubin strongly overlapped with the emission spectrum of $\mathrm{MoS}_{2}$ QDs, and the distance between $\mathrm{MoS}_{2}$ QDs and bilirubin was calculated to be $4.19 \mathrm{~nm}$ [47]. The level of fluorescence quenching was linearly related to the concentration of bilirubin in the range of $0.5-25 \mu \mathrm{m}$. The sensor exhibited acceptable specificity toward bilirubin and was successfully applied in the real sample analysis.

In cases that the target affects the FRET process, the target could either enhance the efficiency of energy transfer $[48,49]$ or inhibit the FRET process [49-55]. Yu et al. [48] found that when the distance between the donor $\left(\mathrm{MoS}_{2}\right.$ QDs) and the acceptor (FAM) was excessively short because of the adsorption of FAM-labeled molecular beacons (MBs) on $\mathrm{MoS}_{2}$ QDs, the FRET efficiency was relatively low. However, after the addition of target miRNA, which could trigger the opening of hairpins, the distance between $\mathrm{MoS}_{2}$ QDs and FAM was enlarged and more suitable for the occurrence of FRET, leading to the enhanced fluorescence emission of FAM (Figure 2A). The fluorescence ratio of $\mathrm{MoS}_{2}$ QDs and FAM was inversely related to the concentration of
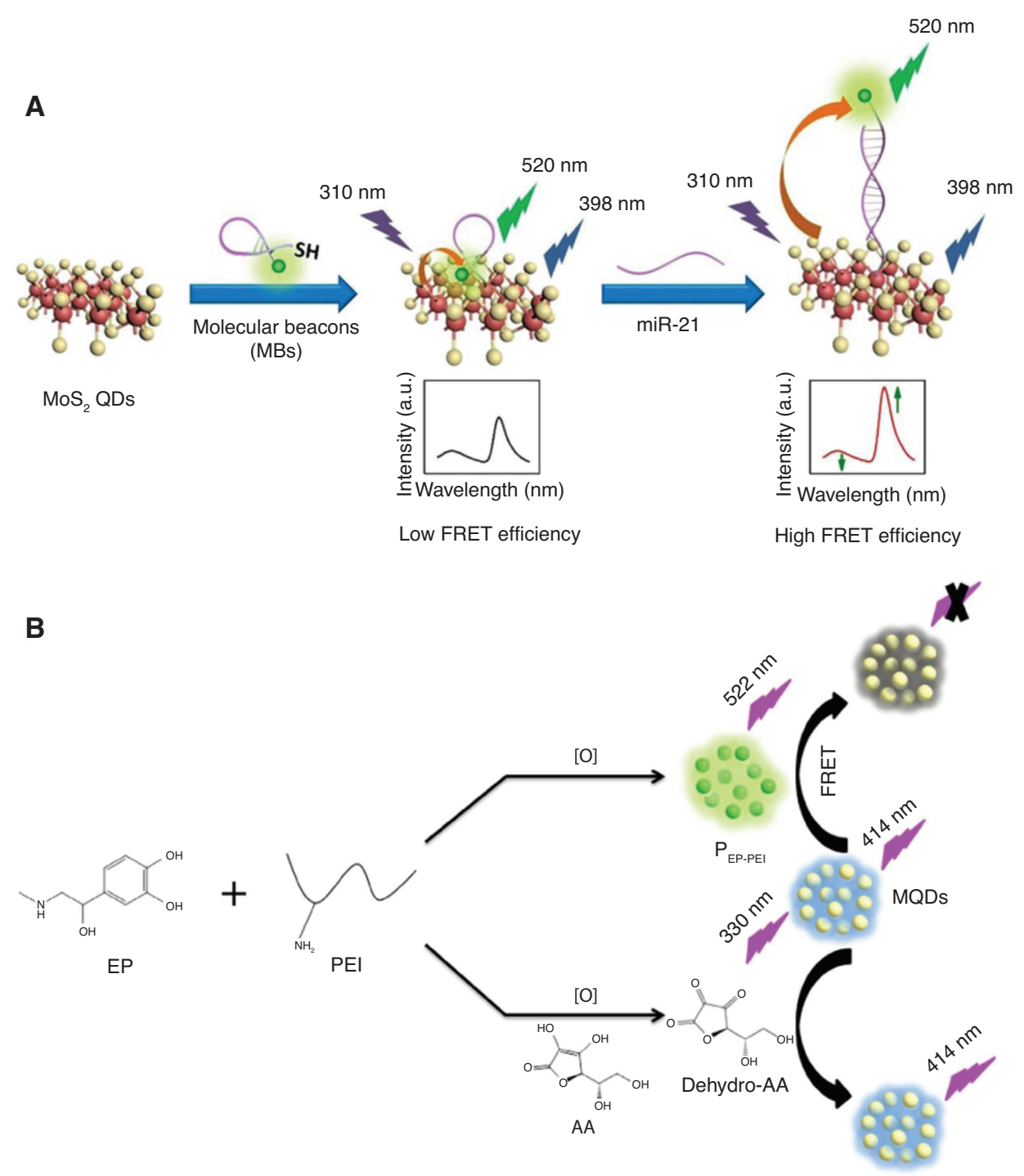

Figure 2: Schematic illustration of FRET sensors using $\mathrm{MOS}_{2}$ QDs as the donor.

(A) Schematic of the MoS2 QD@MB nanoprobe-based FRET sensor for miRNA detection. Reprinted with permission from Yu et al. [48]. Copyright (2018) Springer. (B) Schematic representation of the MoS2 QD-based FRET sensor for the detection of EP and AA. Reprinted with permission from Zhang et al. [49]. Copyright (2019) Elsevier. 
miRNA in the range of 5-150 nM. Then, sensor could detect as low as 0.38 nm miRNA. The MoS 2 QDs@FAM-MB nanoprobe could also be used to determine whether the cells express the target miRNA. Ascorbic acid (AA) and epinephrine (EP) are both important biomarkers for some diseases. Zhang et al. [49] developed a FRET system for simultaneous detection of EP and AA. In the system, the effects of $\mathrm{EP}$ and $\mathrm{AA}$ were opposite. As shown in Figure 2B, the EP underwent the polymerization process in the polyethyleneimine (PEI) solution and formed a fluorescent copolymer of $\mathrm{P}_{\text {EP-PEI }}$ As the absorption band of $\mathrm{P}_{\text {EP-PEI }}$ effectively overlapped with the emission band of $\mathrm{MoS}_{2}$ QDs, the fluorescence of $\mathrm{MoS}_{2} \mathrm{QDs}$ was quenched. At the same time, the fluorescence of $\mathrm{P}_{\mathrm{EP} \text {-PEI }}$ was enhanced because of the energy transfer. However, the AA, which inhibited the oxidative polymerization of EP, would suppress the formation of $\mathrm{P}_{\mathrm{EP}}$ ${ }_{\text {PEI }}$ and the FRET effect. As a result, the fluorescence of $\mathrm{MOS}_{2}$ QDs recovered, and the fluorescence of $\mathrm{P}_{\mathrm{EP} \text {-PEI }}$ reduced. By measuring the fluorescence ratio of $\mathrm{MoS}_{2}$ QDs and $\mathrm{P}_{\text {EP-PEI}}$, the limit of detection (LOD) of EP and AA was achieved as low as 0.05 and $0.2 \mu \mathrm{M}$, respectively.

$\mathrm{WS}_{2}$ QDs, another kind of TMDs QDs, were also employed as the donor in FRET sensors [56]. The $\mathrm{WS}_{2}$ dots synthesized by hydrothermal method showed a high quantum yield of 0.066 at the excitation of $324 \mathrm{~nm}$. Nitrofurazone (NFZ), a threatening chemical to human health, was found to have an absorption band that largely overlapped with the emission band of $\mathrm{WS}_{2}$ QDs. Thus, the authors utilized the $\mathrm{WS}_{2}$ QDs: NFZ as the donor-acceptor pair for NFZ detection. After the addition of NFZ to the $\mathrm{WS}_{2}$ QDs solution, the fluorescence emission of $\mathrm{WS}_{2}$ QDs at $405 \mathrm{~nm}$ gradually decreased with the increment of NFZ concentration in the solution. The developed sensor exhibited a dynamic range of 0.17-166 $\mu \mathrm{m}$ with a detection limit of $0.055 \mu \mathrm{M}$.

Two-dimensional BP, the first and mostly explored group VA 2D nanomaterial, was first synthesized by the mechanical exfoliation from bulk black phosphorus in $\alpha$-phase [64]. Similar to graphene, each layer of BP is in hexagonal structure and possesses two in-plane directions, that is, zigzag and armchair direction. However, BP exhibits high anisotropy in electrical, optical, and thermal properties along the two directions because of its low in-plane symmetry [65]. Black phosphorene QDs were first prepared by Zhang et al. [66] in 2015 by liquidphase ultrasonic exfoliation. Black phosphorene QDs could emit strong blue or green fluorescence that depends on the excitation wavelength and have been utilized in the fluorescence sensing by researchers [57, 67, 68]. Ying et al. employed the BP QDs as nanofluorophores for the FRET sensing of DNA [57]. The BP QDs were prepared by the sonication exfoliation method. The resultant BP
QDs emitted fluorescence at $527 \mathrm{~nm}$ with the irradiation of 200-nm light. Similar to our research [69], the authors utilized the different affinities of ssDNA and dsDNA to BP QDs. In the absence of target DNA, the quencher, dabcyl, would quench the fluorescence of the BP QDs because of the adsorption of dabcyl-labeled ssDNA on BP QDs. However, after the addition of target DNA, the DNA would be released from the BP QDs after the formation of dsDNA complex. As a result, the fluorescence of BP QDs could recover. The sensor exhibited an extremely low LOD of $5.9 \mathrm{p} \mathrm{pM}$ and an appreciable linearity in the range of 4-4000 pM for the target DNA.

\subsection{Other 2D nanomaterials as the donor in FRET sensors}

As mentioned in the introduction, some 2D nanomaterials have intrinsic fluorescence owing to their unique molecule structure. For instance, 2D g- $\mathrm{C}_{3} \mathrm{~N}_{4}$ nanosheets exhibit strong PL due to the existence of condensed tri-s-triazine units, which can act as the donor in FRET sensors. Zhang et al. [26] developed a FRET system, in which the g- $\mathrm{C}_{3} \mathrm{~N}_{4}$ nanosheets acted as the donor, and the $\mathrm{MnO}_{2}$ nanosheets acted as the quencher for intracellular GHS imaging. The $\mathrm{MnO}_{2}$ nanosheets were able to grow in situ on both sides of $\mathrm{g}-\mathrm{C}_{3} \mathrm{~N}_{4}$ nanosheets to form a sandwich composite as the manganese ions could be readily adsorbed on the $\mathrm{g}-\mathrm{C}_{3} \mathrm{~N}_{4}$ nanosheets with abundant functional groups. As a result, the fluorescence of $\mathrm{g}-\mathrm{C}_{3} \mathrm{~N}_{4}$ nanosheets was quenched by $\mathrm{MnO}_{2}$ through the FRET. After the addition of glutathione (GSH), the $\mathrm{MnO}_{2}$ would be reduced to $\mathrm{Mn}^{2+}$ and released from the $\mathrm{g}-\mathrm{C}_{3} \mathrm{~N}_{4}$ nanosheets, leading to the fluorescence recovery of $\mathrm{g}-\mathrm{C}_{3} \mathrm{~N}_{4}$ nanosheets (Figure $3 \mathrm{~A}$ ). The authors incubated the nanocomposite with HeLa cells. As shown in Figure 3B, the nanocomposite exhibited blue fluorescence inside the cells after $2 \mathrm{~h}$ of incubation, indicating the successful delivery of nanocomposite into the cytoplasm and the expression of GSH in the HeLa cells. However, when the cells were pretreated with $\mathrm{N}$-methylmaleimide (NMM), the GSH expressed by the cells would be reduced by NMM, resulting in a decrease of the GSH level. Thus, despite that the fluorescence of g- $\mathrm{C}_{3} \mathrm{~N}_{4}$ nanosheets was still quenched by the $\mathrm{MnO}_{2}$, the cells could not show obvious fluorescence. Other groups have also utilized the $\mathrm{g}-\mathrm{C}_{3} \mathrm{~N}_{4}$ nanosheet $-\mathrm{MnO}_{2}$ sandwich nanocomposite for the detection of ricin [59], $\mathrm{H}_{2} \mathrm{O}_{2}$, and glucose [58]. Men et al. [59] innovatively constructed a sandwich nanocomposite-based FRET system for ultrasensitive detection of ricin, in which the liposome was used for amplification. As shown in Figure 3C, in the presence of ricin, the liposome would be released from the 


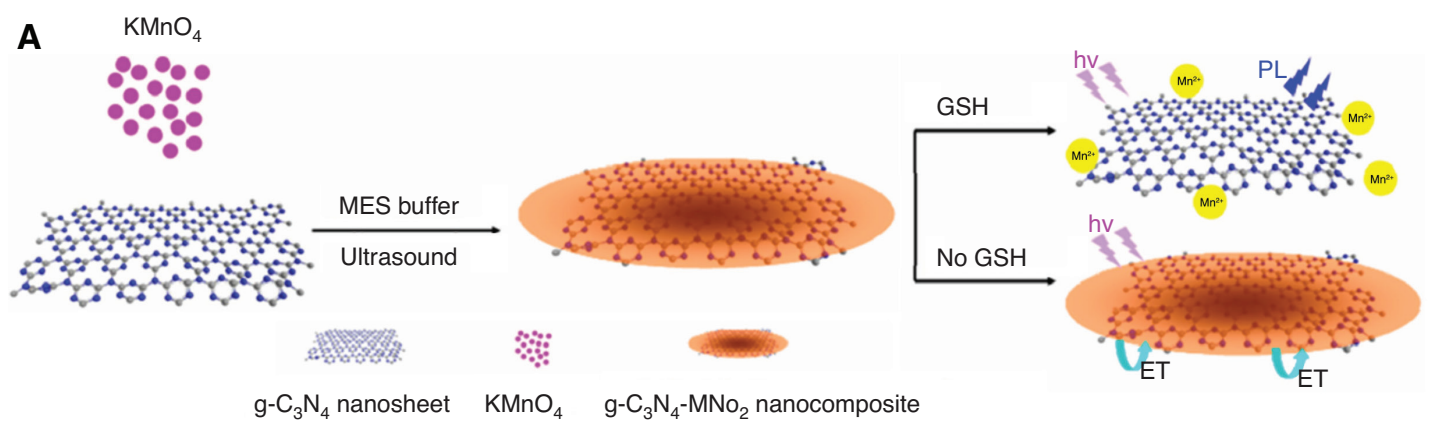

B

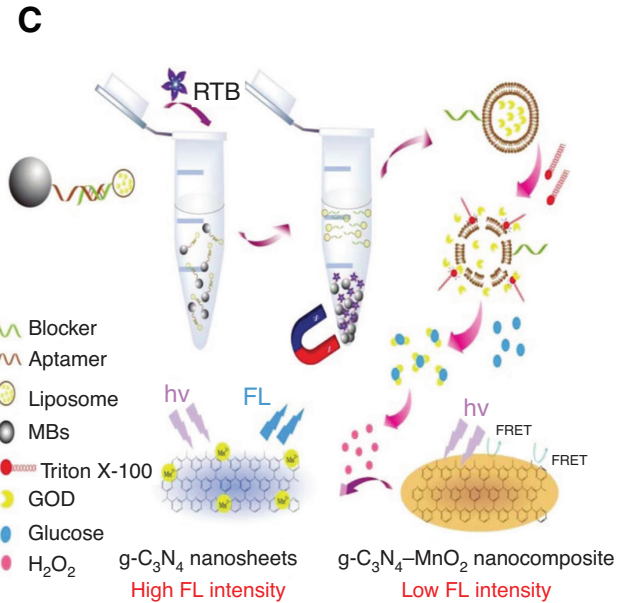

Figure 3: Schematic illustration and experimental results of FRET sensors using $\mathrm{g}-\mathrm{C}_{3} \mathrm{~N}_{4}-\mathrm{MnO}_{2}$ nanocomposite as the donor-acceptor pair. (A) GSH sensing mechanism of $\mathrm{g}-\mathrm{C}_{3} \mathrm{~N}_{4}-\mathrm{MnO}_{2}$ nanocomposite-based FRET sensor. (B) Fluorescence microscopy images (top row) and the overlapping images (bottom row) of HeLa cells: (a) control cells, (b) cells incubated with the $\mathrm{g}-\mathrm{C}_{3} \mathrm{~N}_{4}-\mathrm{MnO}_{2}$ nanocomposite, (c) cells pretreated with NMM for $20 \mathrm{~min}$, followed by $\mathrm{g}-\mathrm{C}_{3} \mathrm{~N}_{4}-\mathrm{MnO}_{2}$ nanocomposite. Scale bar $=50 \mu \mathrm{m}$. Reprinted with permission from Zhang et al. [26]. Copyright (2014) American Chemical Society. (C) RTB detection mechanism of g- $\mathrm{C}_{3} \mathrm{~N}_{4}-\mathrm{MnO}_{2}$ nanocomposite and liposome-based FRET sensor. Reprinted with permission from Men et al. [59]. Copyright (2018) The Royal Society of Chemistry.

magnetic beads due to the hybridization of ricin and its aptamer. After the separation of magnetic beads, the free liposome was broken by Triton X-100, and the encapsulated glucose oxidase leaked out from the liposome. The glucose oxidase then catalyzed the oxidation of glucose and generated $\mathrm{H}_{2} \mathrm{O}_{2}$, which subsequently reduced the $\mathrm{MnO}_{2}$ to $\mathrm{Mn}^{2+}$ and blocked the FRET process of the sandwich nanocomposite. As a liposome could load a large amount of glucose oxidase, the fluorescence recovery of g- $\mathrm{C}_{3} \mathrm{~N}_{4}$ nanosheets could be amplified. Under optimal conditions, the sensor could detect as low as $190 \mathrm{ng} / \mathrm{ml}$ ricin. The authors also verified the capability of the sensor to detect the ricin concentration in real samples.

Like 2D-QDs, the $\mathrm{g}-\mathrm{C}_{3} \mathrm{~N}_{4}$ nanosheets have also been employed in a FRET system as the donor for direct sensing of quenchers [60-62]. Similarly, the absorption spectrum of quenchers should overlap with the emission spectrum of $\mathrm{g}-\mathrm{C}_{3} \mathrm{~N}_{4}$ nanosheets. The $\mathrm{g}-\mathrm{C}_{3} \mathrm{~N}_{4}$ nanosheets could also provide abundant functional groups (e.g. carboxyl and amino groups) for the adsorption of quenchers or acceptors. Guo et al. prepared the $\mathrm{g}-\mathrm{C}_{3} \mathrm{~N}_{4}$-gold NCs (AuNCs) nanocomposite by the covalent binding of $\mathrm{g}-\mathrm{C}_{3} \mathrm{~N}_{4}$ and BSA-capped AuNCs [62]. The nanocomposite emitted a strong fluorescence at $420 \mathrm{~nm}$ under the 320-nm excitation. When the dopamine was added to the nanocomposite, the reduction product of dopamine, dopamine quinine, would adsorb on the surface of the nanocomposite and quench the fluorescence of nanocomposite as the absorption spectrum of dopamine quinine overlapped with the emission spectrum of nanocomposite. Compared with the $\mathrm{g}_{-} \mathrm{C}_{3} \mathrm{~N}_{4}$, the nanocomposite-based FRET sensor exhibited a better sensitivity toward dopamine detection.

The 2D organic-inorganic perovskite features a typical structure on which the inorganic and organic layers alternatively grow on each other [70, 71]. The organic constituents enable the perovskite good plasticity, structural flexibility, and high fluorescence quantum yield, and the inorganic components ensure the perovskite more stable mechanical strength and thermal stability. The exceptional optical properties of perovskite have been employed in the optical sensing applications [29, 72]. Huang et al. [29] developed a novel FRET-based sensor for $\mathrm{Hg}^{2+}$ detection by using the 2D 
organic-inorganic perovskite as the energy donor. Initially, the fluorescence emission of synthetic 2D perovskite, $\mathrm{C}_{10} \mathrm{PbBrPE}$, at $550 \mathrm{~nm}$ was not quenched by the nonfluorescent rhodamine B ethylenediamine (RBEA). Upon the addition of $\mathrm{Hg}^{2+}$, the RBEA coordinated with $\mathrm{Hg}^{2+}$ and underwent the ring-open process, resulting in a fluorescent complex, of which the absorption band overlapped with the emission band of perovskite. As the RBEA was strongly attached to the perovskite through hydrogen bonding, the FRET process would happen between the RBEA- $\mathrm{Hg}^{2+}$ and perovskite. Consequently, the fluorescence emission intensity of perovskite gradually decreased, and the fluorescence emission intensity of RBEA- $\mathrm{Hg}^{2+}$ increased with the increasing concentration of $\mathrm{Hg}^{2+}$. The sensor could detect as low as $2.36 \mu \mathrm{M}$ $\mathrm{Hg}^{2+}$ with acceptable specificity.

As a summary, both the 2Q-QDs and the 2D nanomaterials that own inherent PL have been successfully employed as donors in the FRET sensors. In the development of FRET sensors, the detection targets could act as either the energy acceptor or the substance affecting the FRET process. When the target acts as the energy acceptor, the absorption spectrum of target should strongly overlap with the emission spectrum of the donor. And the target should be adsorbed on the surface of the 2D nanomaterials so that the distance between the acceptor and the donor could be short enough for the FRET to occur. In cases that the target affects the FRET process, the interaction of the targets and probe molecules could either enhance the efficiency of energy transfer or inhibit the FRET process. Compared with traditional fluorescent nanomaterials, the 2D nanomaterials exhibit much better adsorption ability toward molecules owing to the $\pi-\pi$ stacking interaction and the abundant chemical groups on the surface of $2 \mathrm{D}$ nanomaterials. As a result, the FRET sensor is more easily to be fabricated.

\section{2D nanomaterials as the acceptor in FRET sensors}

\section{1 $\mathrm{MoS}_{2}$ nanosheets as the acceptor in FRET sensors}

Transition metal dichalcogenide nanosheets are generally employed as fluorescence quenchers in FRET sensors for biomedical analysis because of their fast electron transfer rate and strong optical absorption. The fluorescence-quenching property of $\mathrm{MoS}_{2}$ is the most commonly exploited in the FRET sensors compared with other TMDs. In 2013, for the first time, Zhu et al. [73] developed a FRET sensor for the detection of DNA and small molecules by using monolayer $\mathrm{MoS}_{2}$ as the fluorescence quencher (Figure 4A). It was found that $98 \%$ of fluorescence emission was quenched within 5 min after the mixing of dyelabeled ssDNA and $\mathrm{MoS}_{2}$, verifying the strong biomolecule adsorption and fluorescence-quenching capability of $\mathrm{MoS}_{2}$. However, the $\mathrm{MoS}_{2}$ exhibited weak affinities to the dsDNA due to the buried nucleobases, resulting in the recovery of fluorescence (Figure 4B). The level of fluorescence recovery depended on the concentration of target DNA. In another experiment, the dye-labeled antiadenosine aptamer and $\mathrm{MoS}_{2}$ were used for the detection of adenosine (Figure 4C). The interaction of adenosine and aptamer could induce structural transformation of the DNA probe, which further prevented the adsorption of aptamer on $\mathrm{MoS}_{2}$. The LOD of the $\mathrm{MoS}_{2}$-based assay was calculated to be $500 \mathrm{pM}$ and $5 \mu \mathrm{M}$ for DNA and adenosine, respectively. Since then, dozens of researchers have reported the development of $\mathrm{MoS}_{2}$-DNA-based FRET sensors for the detection of various targets, such as the nucleic acids [74-85], proteins [86-95], metal ions [96, 97], small molecules [52, 86, 98-104], enzyme activity [105],
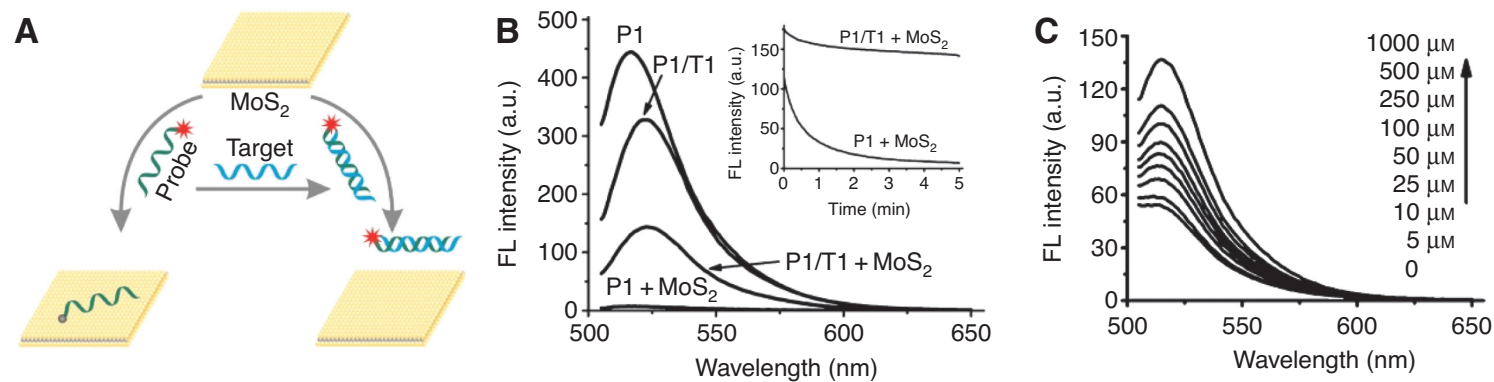

Figure 4: Schematic representation and experimental results of $\mathrm{MoS}_{2}$-based FRET sensor for DNA detection.

(A) Schematic representation of the $\mathrm{MoS}_{2}$-based FRET sensor for DNA detection. (B) Fluorescence spectra of ssDNA and DNA duplex mixed with and without $\mathrm{MoS}_{2}$. Inset, Real-time fluorescence response of ssDNA and DNA duplex reacted with $\operatorname{MoS}_{2}$. (C) Fluorescence spectra of the FAM-labeled aptamer reacted with different concentrations of adenosine and then mixed with $\mathrm{MoS}_{2 .}$ Reprinted with permission from Zhu et al. [73]. Copyright (2013) American Chemical Society. 
and others [106]. These sensors mainly take advantage of the fluorescence-quenching capability and different affinity of $\mathrm{MoS}_{2}$ to ssDNA and ssDNA-target combination, enabling easy preparation, single-step, and rapid detection.

The fluorescent donor in a $\mathrm{MoS}_{2}$-based FRET sensor could be dyes [73, 75-91, 95, 96, 98, 102, 104-107], carbon dots [97, 100, 101, 108], UCNPs [109], silver NCs [99, 103], QDs [52, 82, 92, 94], and AuNCs [93]. The fluorescence emission wavelength of these donors ranges from 360 to $680 \mathrm{~nm}$, which validates the broad-spectrum quenching property of $\mathrm{MoS}_{2}$. However, the $\mathrm{MoS}_{2}$-DNA-based direct sensing suffers from low sensitivity, normally in the nanomolar range. Moreover, the specificity of these sensors is dissatisfactory due to the nonspecific adsorption. For instance, the single-base mismatch DNA would induce half-response of the complementary target DNA at the same concentration [76]. To overcome these issues, various methods for signal amplification $[74,78,80,81$, $83]$ and the sensor specificity enhancement $[80,81,91]$ have been adopted. Kenry et al. [91] put forward a means to enhance the specificity of $\mathrm{MoS}_{2}$-based FRET aptasensor by blocking the $\mathrm{MoS}_{2}$ surface with bovine serum albumin. They found that the fluorescence recovery after the addition of nonspecific protein was greatly suppressed under blocking treatment. They hypothesized that the nonspecific fluorescence recovery was primarily caused by nonspecific protein adsorption, which displaced the binding sites of labeled aptamer. Xiao et al. [80, 81] utilized MBs probe and duplex-specific nuclease (DSN) to achieve highly sensitive and selective detection of microRNAs. As shown in Figure 5A, the polyC-MBs were first adsorbed on $\mathrm{MoS}_{2}$ via the VdW forces between $\mathrm{MoS}_{2}$ and polyC, leading to the fluorescence quenching. The MB block was arranged on the $\mathrm{MoS}_{2}$ surface to recognize its complementary target. After polyC-MB hybridized to its microRNA target, a DNA/RNA heteroduplex was formed and then became the digested target for DSN. As a result, the MBs were cleaved into short oligonucleotides, and

A

microRNA $\frown$

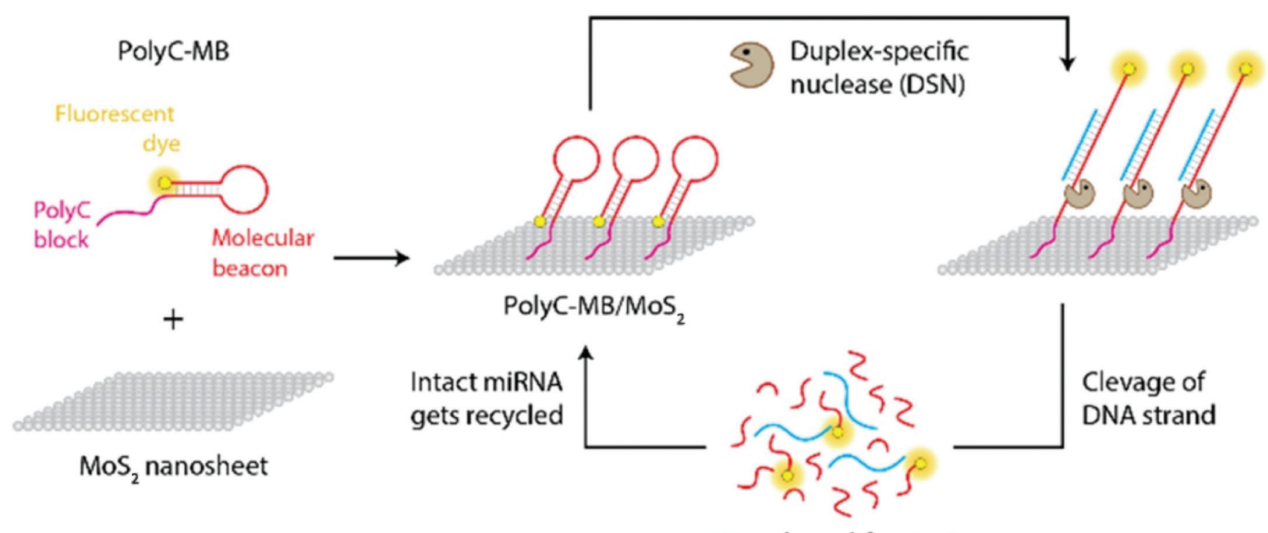

B

[Signal amplification]

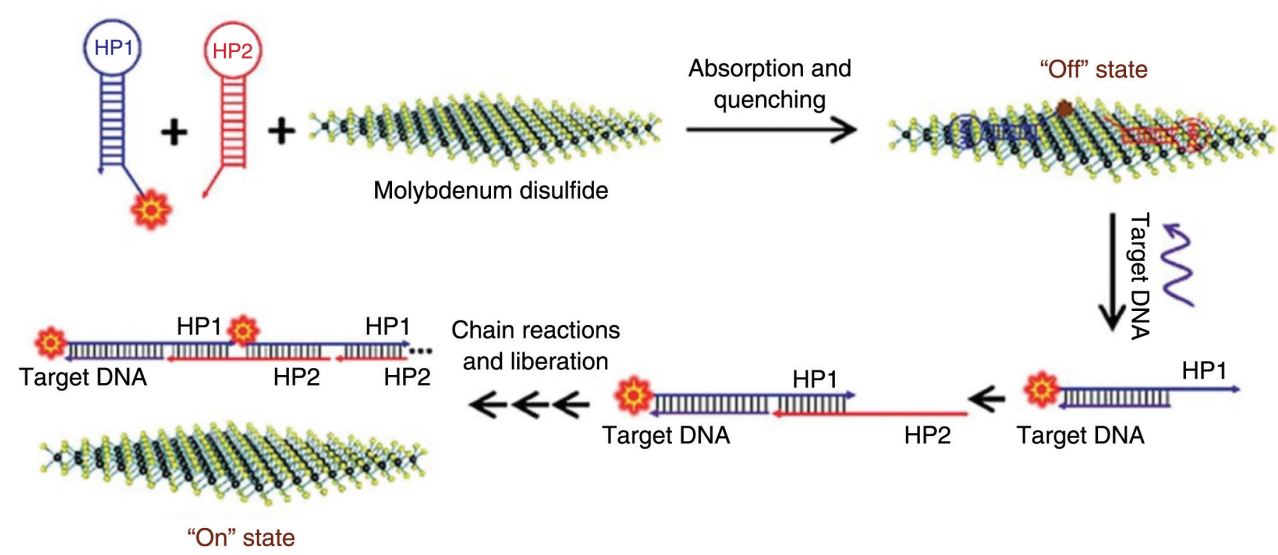

Figure 5: Schematic illustration of FRET sensors using signal amplification strategy.

(A) Schematic diagram of an ultrasensitive microRNA assay based on MBs probe and DSN. Reprinted with permission from Xiao et al. [80]. Copyright (2018) American Chemical Society. (B) Detection principle of the HCRs-amplified FRET DNA assay. Reprinted with permission from Huang et al. [74]. Copyright (2015) The Royal Society of Chemistry. 
the fluorescence was recovered owing to the weak affinity between short oligonucleotides and $\mathrm{MoS}_{2}$. However, the target microRNAs were released from the heteroduplex and then hybridized to another PolyC-MB, initiating the next cycle of reactions. Thanks to the DSN-mediated signal amplification, the sensor exhibited an ultrahigh sensitivity toward microRNA ( $\mathrm{LOD}=3.4 \mathrm{fM}$ ). Additionally, the remarkable selectivity of MBs and DSN enabled the sensor to precisely distinguish microRNAs with one-base mismatch. Huang et al. [74] reported a signal amplification method using target-triggered hybridization chain reactions (HCRs). As shown in Figure 5B, HP1 and HP2, two complementary hairpin DNA probes, were first adsorbed on the $\mathrm{MoS}_{2}$ surface, and the fluorescence of HP1 was quenched by $\mathrm{MoS}_{2}$. Subsequently, the hairpin of $\mathrm{H} 1$ was opened by the hybridization with target DNA, thus inducing the HCRs between $\mathrm{H} 1$ and H2. Consequently, the fluorescence of HP1 recovered. The sensor could detect as low as 15 pM target DNA. Other researchers have exploited the exonuclease-assisted amplification for sensitive detection of nucleic acid [78, 83].

Besides the $\mathrm{MoS}_{2}$-DNA-based FRET sensors that utilize the strong affinity of $\mathrm{MoS}_{2}$ to ssDNA, the $\mathrm{MoS}_{2}$ could also be modified with functional molecules to achieve the affinity toward other probe molecules. Xuan and Neethirajan exploited the poly(L-lysine) (PLL)-functionalized $\mathrm{MoS}_{2}$ $\left(\mathrm{PLL}-\mathrm{MoS}_{2}\right.$ ) to implement the binding of infectious bronchitis virus (IBV) antibody (Ab) on $\mathrm{MoS}_{2}$ [110]. In the presence of IBV, the dyed-IBV-Ab, IBV, and the $\mathrm{MoS}_{2}-\mathrm{IBV}-\mathrm{Ab}$ formed a sandwich structure due to the immune reaction (Figure 6A). Consequently, the fluorescence of the dyedIBV-Ab probe was quenched because of the FRET between the closely connected dyes and $\mathrm{MoS}_{2}$. The LOD for IBV was $4.6 \times 10^{2} \mathrm{EID}_{50} / \mathrm{ml}$ in phosphate-buffered saline, and the sensor exhibited good recovery and consistency toward the spiked IBV. Yang et al. [94] developed a 4-mercaptophenylboronic acid-functionalized $\mathrm{MoS}_{2}\left(\mathrm{PBA}-\mathrm{MoS}_{2}\right)$ nanosheets-based FRET sensor for simultaneous detection of multiple lectins. Initially, the saccharide-functionalized CdSe/ZnS QDs (s-QDs) were adsorbed on the nanosheets through the chemical interaction between PBA and saccharide, and the fluorescence of s-QDs was quenched by $\mathrm{MoS}_{2}$. The competitive binding of lectins to s-QDs induced the dissociation of s-QDs from $\mathrm{MoS}_{2}$, and then the fluorescence of s-QDs was restored (Figure $6 \mathrm{~B})$. As the affinity of lectin to different saccharides was
A
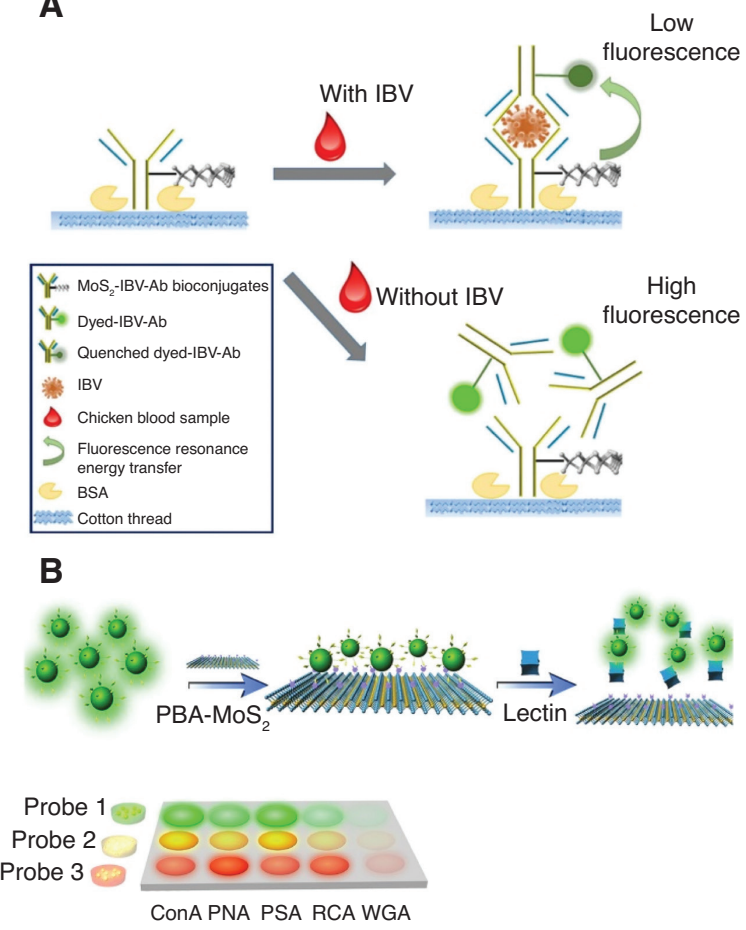

C

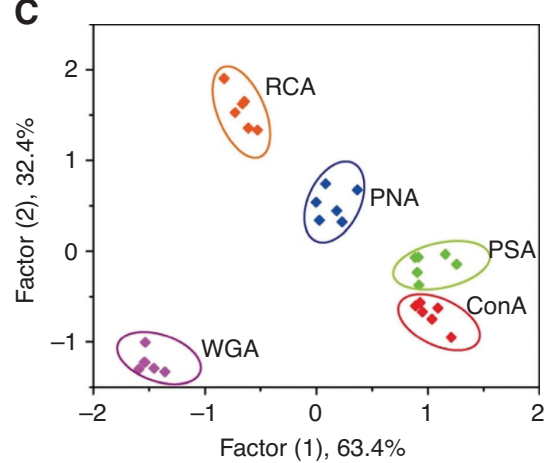

D

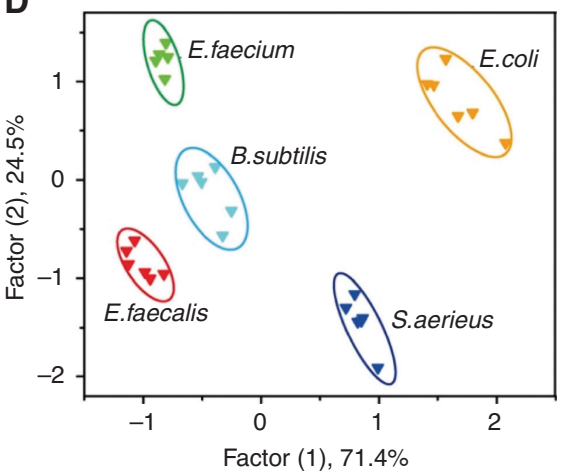

Figure 6: Detection principle and experimental results of $\mathrm{MOS}_{2}$-based FRET biosensors for virus and bacteria detection. (A) Detection principle of a FRET platform for IBV detection. Reprinted with permission from Xuan and Neethirajan [110]. Copyright (2018) IEEE. (B) Experimental design of a PBA-MoS 2 -based FRET biosensor for lectin detection. (C) Canonical score plot of lectins via linear discriminant analysis signal patterns. (D) Canonical score plots of bacteria via LDA signal patterns. Reprinted with permission from Yang et al. [94]. Copyright (2018) Springer. 

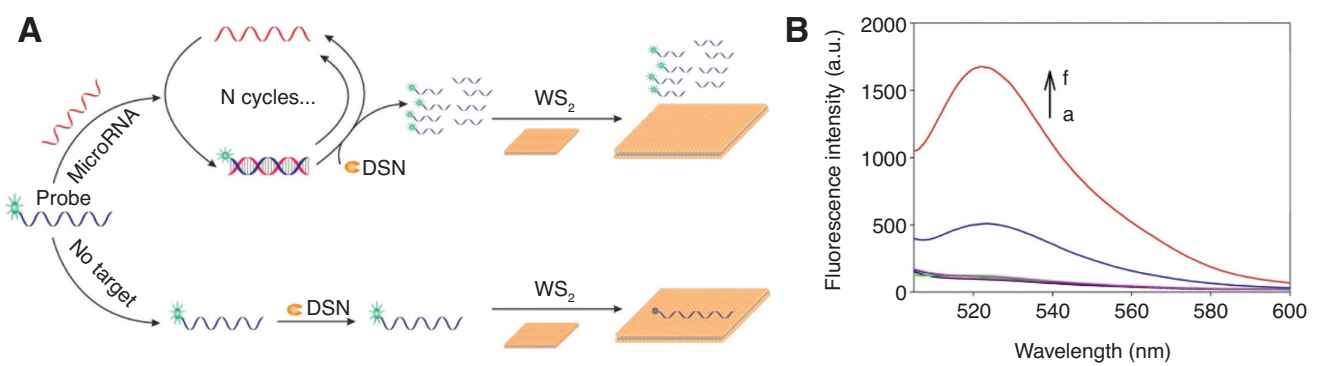

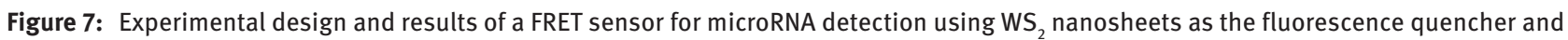
DSN for signal amplification.

(A) Experimental design of a microRNA assay by using $\mathrm{WS}_{2}$ nanosheets as the fluorescence quencher and DSN for signal amplification.

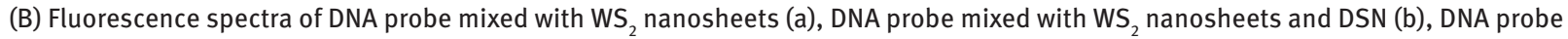
mixed with mismatched microRNA and WS ${ }_{2}$ nanosheets (c), DNA probe mixed with mismatched microRNA, WS ${ }_{2}$ nanosheets and DSN (d), DNA probe mixed with target microRNA and WS $_{2}$ nanosheets (e), and DNA probe mixed target microRNA, WS ${ }_{2}$ nanosheets and DSN (f). Reprinted with permission from Xi et al. [111]. Copyright (2014) American Chemical Society.

diverse, the fluorescence recovery degree of three s-QDs would be different as the lectins coexisted. Accordingly, multiple lectins and bacteria were accurately identified with $100 \%$ accuracy by recognizing the distinct fluorescence response patterns (Figure 6C, D).

\section{2 $\mathrm{WS}_{2}$ nanosheets as the acceptor in FRET sensors}

Apart from $\mathrm{MoS}_{2}, \mathrm{WS}_{2}$ could also act as an efficient quencher for fluorescence donors [85, 111-120]. Xi et al. [111] reported for the first time that the $\mathrm{WS}_{2}$ nanosheets were utilized to develop a FRET sensor for the detection of microRNA. Figure 7A shows that the formed heteroduplex by the hybridization of probe ssDNA and target microRNA became the target of DSN cleavage, implementing the cleavage of probe DNA into short fragments and the releasing of target microRNA for new cycle of reactions. Upon the mixing of $\mathrm{WS}_{2}$, the product from the DSNSA reaction and the uncut probe DNA could be easily distinguished by the recorded fluorescence signal owing to the differential affinity of $\mathrm{WS}_{2}$ for short oligonucleotide fragment ( $<10$ bases) and probe ssDNA ( $>10$ bases) (Figure $7 \mathrm{~B})$. Both the effective fluorescence-quenching capacity of $\mathrm{WS}_{2}$ and DNS-assisted signal amplification ensured the ultrasensitivity and high specificity of the biosensor.

In several studies, the performance of $\mathrm{MoS}_{2}$ and $\mathrm{WS}_{2}$ in FRET biosensors was compared [77, 85, 118]. Loo et al. [77] investigated the performance of $\mathrm{MoS}_{2}$ and $\mathrm{WS}_{2}$ for the fluorescent detection of nucleic acids. The results showed that the fluorescence-quenching efficiency of $\mathrm{MoS}_{2}$ and $\mathrm{WS}_{2}$ was similar at the optimal amount of $\mathrm{MoS}_{2}$ and $\mathrm{WS}_{2}$. However, $\mathrm{WS}_{2}$ led to more fluorescence retention than $\mathrm{MoS}_{2}$ after the addition of the same amount of complementary DNA target, indicating the weaker interactions between $\mathrm{WS}_{2}$ and DNA. As a result, $\mathrm{MoS}_{2}$ presented wider dynamic range and higher selectivity than $\mathrm{WS}_{2}$. However, in two other reports, the $\mathrm{MoS}_{2}$ and $\mathrm{WS}_{2}$ nanosheets showed similar performance in the detection of collagen sequence [118] and the progress monitoring of polymerase chain reactions [85].

\section{3 $\mathrm{MnO}_{2}$ nanosheets as the acceptor in FRET sensors}

As discussed above, most TMD-based FRET sensors exploit the differential affinity of TMDs toward probes and the probe-target combination. Unlike other TMDs, the $\mathrm{MnO}_{2}$ nanosheets are chemically active and could be reduced into $\mathrm{Mn}^{2+}$, which makes them good candidates in constructing novel FRET sensors for the detection of reductants, especially GSH, the most abundant thiolated tripeptide in living cells [38, 121-136]. The first study was carried out by Deng et al [38]. Initially, the $\mathrm{MnO}_{2}$ nanosheets were growing on the surface of $\mathrm{NaYF}_{4}: \mathrm{Yb} / \mathrm{Tm} @$ $\mathrm{NaYF}_{4}$ UCNPs by the reduction of $\mathrm{KMnO}_{4}$ in the presence of 2-(N-morpholino) ethanesulfonic acid buffer. Upon NIR excitation, the fluorescence emission of the UCNPs was quenched by the $\mathrm{MnO}_{2}$ nanosheets due to the FRET between closely spaced UCNPs and $\mathrm{MnO}_{2}$ nanosheets. After the GSH was added, the $\mathrm{MnO}_{2}$ was reduced to $\mathrm{Mn}^{2+}$ and dissociated from the UCNPs, resulting in the recovery of fluorescence emission (Figure 8A, B). The LOD of GSH was calculated to be $0.9 \mu \mathrm{M}$. The authors have also demonstrated the capability of the $\mathrm{MnO}_{2}$-modified UCNPs for monitoring intracellular GSH levels in HepG2 and HeLa cells. It should be noted that the distance between FRET donors and $\mathrm{MnO}_{2}$ was shortened by the direct electrostatic interaction of positively charged donors and negatively charged $\mathrm{MnO}_{2}$, rather than the mediation of the 
A
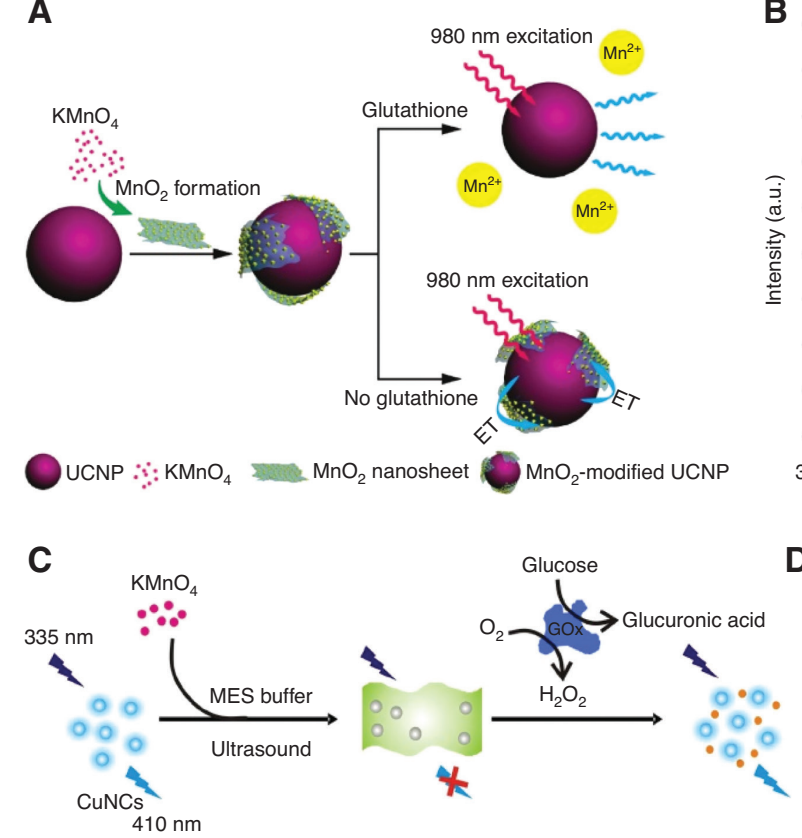

o CuNCs $\mathrm{MnO} \mathrm{Mn}_{2}$ nanosheets $\quad \mathrm{KMnO}_{4} \quad \mathrm{Mn}^{2+}$
B

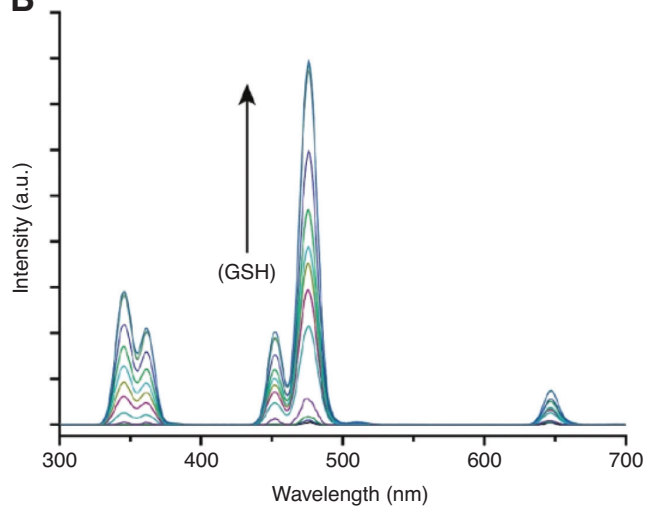

D

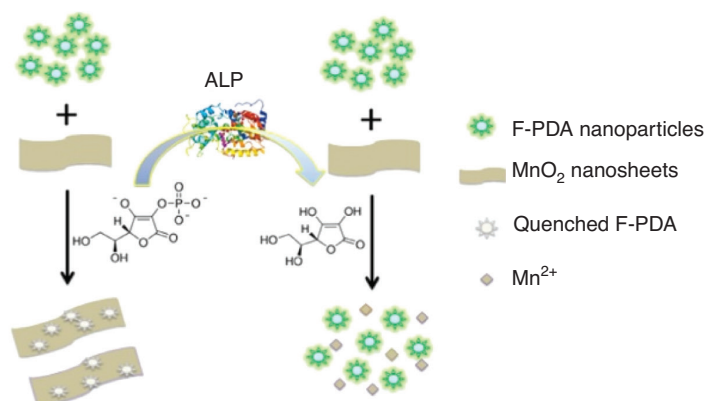

Figure 8: Experimental design and results of FRET sensors using $\mathrm{MnO}_{2}$ nanosheets as the fluorescence quencher. (A) Experimental design of an $\mathrm{MnO}_{2}$-based FRET sensor for $\mathrm{GSH}$ detection. (B) Fluorescence response of $\mathrm{MnO}_{2}$-modified upconversion nanoparticles with the increased GSH concentration from 0 to $10 \mathrm{mM}$. Reprinted with permission from Deng et al. [38]. Copyright (2011) American Chemical Society. (C) Design principle of a FRET glucose assay based on copper nanoclusters-modified $\mathrm{MnO}_{2}$ nanosheets. Reprinted with permission from Wang et al. [137]. Copyright (2016) The Royal Society of Chemistry. (D) Schematic diagram of a FRET ALP bioassay based on fluorescent polydopamine-modified $\mathrm{MnO}_{2}$ probe. Reprinted with permission from reference [138]. Copyright (2018) American Chemical Society.

probe molecules. Since then, dozens of $\mathrm{MnO}_{2}$-based FRET sensors have been developed for GSH sensing in the past 5 years using various fluorescent donors such as persistent luminescence nanoparticles [121], g- $\mathrm{C}_{3} \mathrm{~N}_{4}$ nanosheets [58], QDs [125, 128-132, 135, 136, 139], carbon dots [123, 124, $140]$, gold/copper clusters [126, 133, 137, 141], and two-photon silica nanoparticles [122]. The experimental design of these sensing platforms is the same as that of Deng et al. [38]. The $\mathrm{MnO}_{2} /$ donor composites were first formed by electrostatic interaction or other noncovalent interactions ( $\pi-\pi$ stacking, hydrophobic interaction, etc.), which led to the quenching of fluorescence through FRET. Upon the introduction of $\mathrm{GSH}, \mathrm{MnO}_{2}$ nanosheets were reduced to $\mathrm{Mn}^{2+}$, and thus, the fluorescence of donors recovered. The degree of fluorescence recovery was directly proportional to the GSH concentration. Apart from GSH detection, the $\mathrm{MnO}_{2}$-based FRET sensors have also been applied in the detections of other substances, for instance, AA [139, 142], enzyme activity [138, 139, 141, 143-146], drugs [147], $\mathrm{H}_{2} \mathrm{O}_{2}$ [58, 133], pesticides [140], and glucose [58, 137]. Similar to $\mathrm{GSH}, \mathrm{AA}$ and $\mathrm{H}_{2} \mathrm{O}_{2}$ also present strong reduction capability that could reduce the $\mathrm{MnO}_{2}$ nanosheets into $\mathrm{Mn}^{2+}$.
Moreover, the detection of glucose was achieved by $\mathrm{H}_{2} \mathrm{O}_{2}$ mediated sensing as $\mathrm{H}_{2} \mathrm{O}_{2}$ is one of the oxidation products of the glucose under the catalysis of glucose oxidase [137] (Figure 8C). However, for the detection of enzyme activity, the enzyme could catalyze the hydrolysis of substrate into the product that can reduce $\mathrm{MnO}_{2}$ nanosheets into $\mathrm{Mn}^{2+}$ [138] (Figure 8D). Unlike the studies mentioned above, Garg et al. [147] reported a novel FRET sensor for the detection of anticancer drugs, 6-thioguanine (6-TG) and 6-mercaptopurine (6-MP) based on the fluorescence recovery of carbon dots due to the formation of Mn-S bond.

\subsection{Other 2D nanomaterials as the acceptor in FRET sensors}

The 2D MOF nanosheets have been widely used in the construction of FRET sensors in nanomedicine as they exhibit several advantages over other 2D nanomaterials such as high structural tunability and degradability [148-153]. In the study of Wang et al. [149], the two-color intracellular adenosine imaging in living cells was achieved by using 
the lanthanide-based MOF nanosheets. Two dye (FAM and TAMRA)-labeled ATP aptamers were exploited in the detection. Unexpectedly, the "turn-down" response of fluorescence occurred when FAM-aptamer bound on ATP. The authors hypothesized that the turn-down response may be due to the reattachment of FAM-aptamer on the edge of the MOFs by the electrostatic interaction between negatively charged FAM and $\mathrm{Ln}^{3+}$. However, the fluorescence of positively charged TAMRA recovered after the binding of TAMRA-aptamer on ATP. Thus, the ATP concentration could be determined by the ratio of $\mathrm{F}_{\text {TAMRA }}^{\prime} / \mathrm{F}_{\text {FAM }}^{\prime}$. When cells were incubated with the FAM-aptamer, TAMRA-aptamer, and $\mathrm{MOF}$, the fluorescence color in cells changed from green to red in the progress of the incubation because the generation of ATP led to the turn-down response of FAM and the "turn-on" response of TAMRA (Figure 9). Consequently, the concentration of adenosines in living cells could be monitored in real time. In a later work, the authors further investigated the interaction mechanism of the surface properties of MOFs to the fluorescence quenching of FAM [150]. It was found that the grafted PEI layer on the positively charged MOF surface can block the interaction between FAM and
A

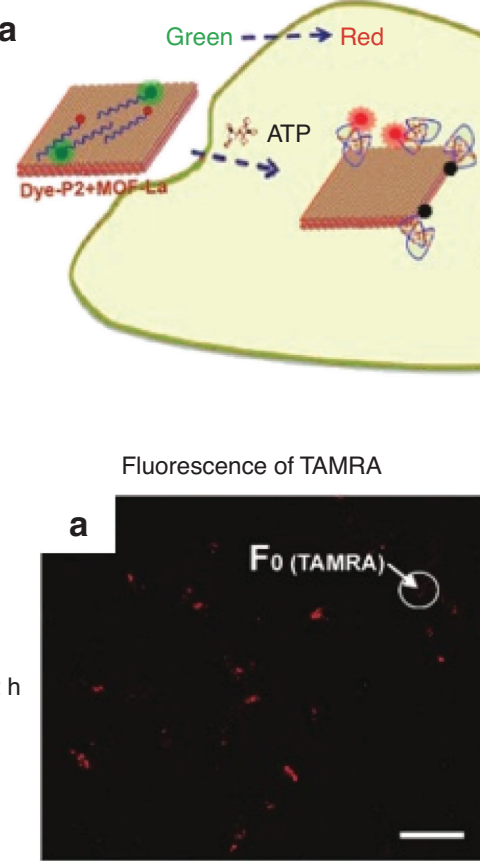

$4 \mathrm{~h}$
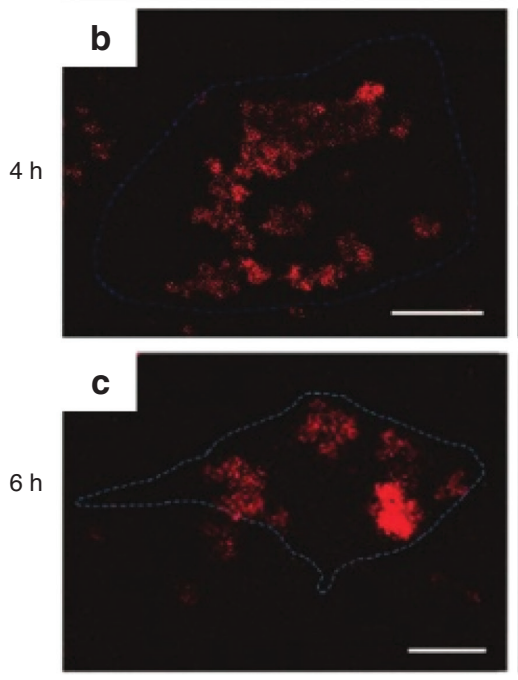

FAM-ATP aptamer mo TAMRA-ATP aptamer Fluorescence of FAM
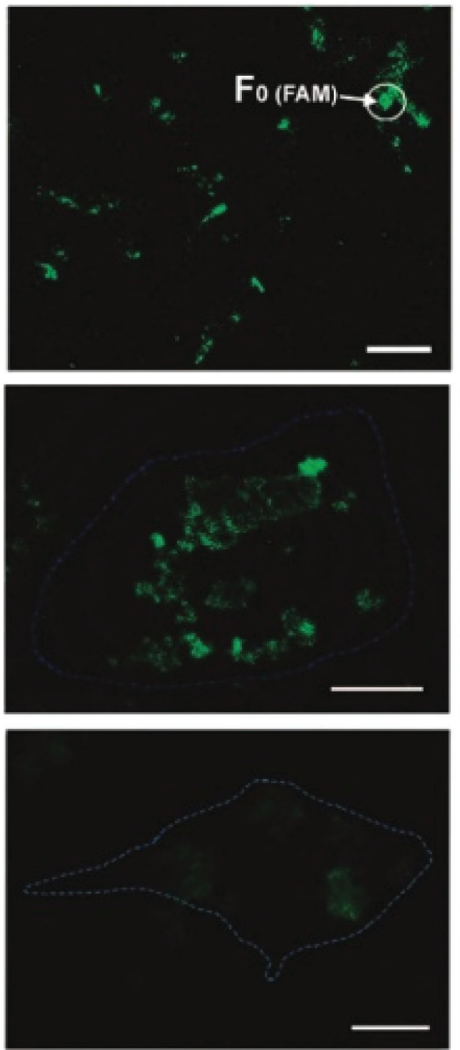

b
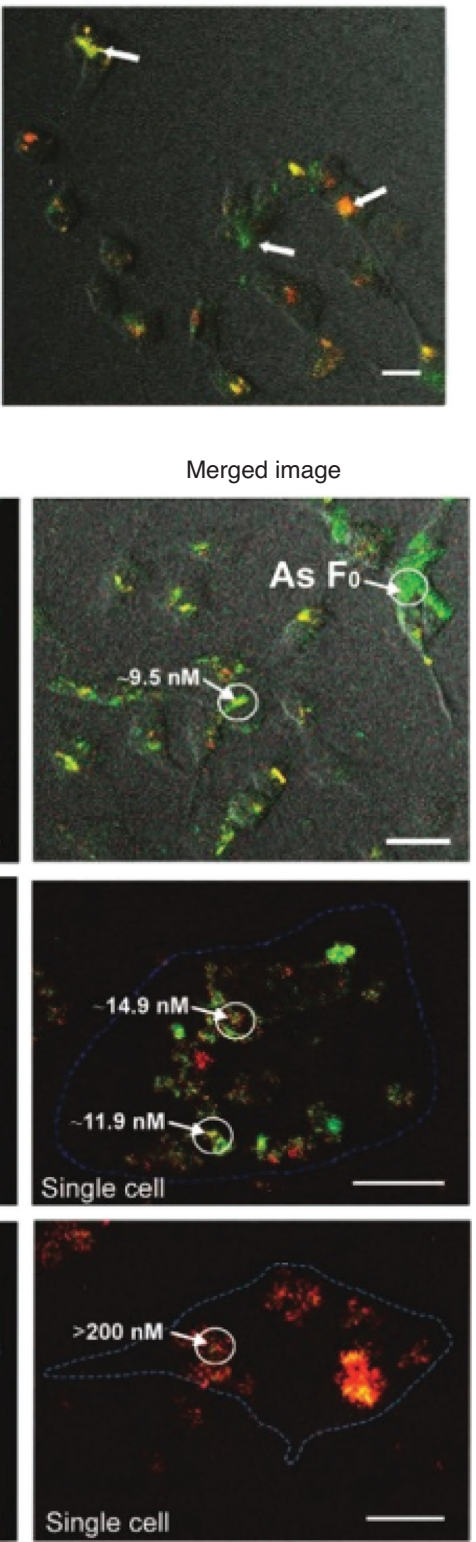

Figure 9: Schematic illustration and experimental results of intracellular adenosine imaging using MOF nanosheets as the fluorescence quencher.

(A) Schematic illustration of intracellular adenosine imaging using FAM-aptamer, TAMRA-aptamer, and MOF nanosheets. Scale bars: 25 um; (B) confocal images of intracellular adenosines and quantification of adenosines concentration at increasing incubation time (2-6 h). Scale bars: a, $30 \mu \mathrm{m}$; b, c, $10 \mu \mathrm{m}$. Reprinted with permission from Wang et al. [149]. Copyright (2017) Springer Nature. 
$\mathrm{Cr}^{3+}$. Thus, the FAM-dsDNA would not be readsorbed on the PEI-MOFs. However, the negatively charged MOFs exhibited similar behaviors toward the FAM-dsDNA compared with the prior study. Gold NPs, graphene, and CNTs, which are normally used as nanoquenchers, exhibited similar fluorescence-quenching properties regardless of the surface charge properties of the nanomaterials. The authors attributed the results to the metal ions on the MOFs that contributed to the unique fluorescence-quenching property toward FAM labeled DNA.

Black phosphorene nanosheets have also been utilized as the quencher in the FRET sensors $[69,154]$. Our group developed a BP nanosheet (BPNSs)-based FRET sensor for microRNA detection [69]. In this study, the molecular dynamics (MD) simulation was employed to prove the differential affinity of BPNSs to ssDNA and DNA/RNA duplex. As shown in Figure 10A, the nucleobases of ssDNA lay flat at the basal plane, whereas the DNA/RNA duplex maintained its helical structure and floated on the surface of the BPNS. The distance between the BPNS and the DNA bases in the duplex exceeded the cutoff of the VdW interactions, demonstrating that the releasing of duplex could be due to the reduced strength of VdW interactions. Under the optimum conditions, the biosensor showed an LOD of $9.37 \mathrm{nM}$ and a dynamic range of 10-1000 nM. In another study, Hu et al. developed a BPNS-based FRET sensor to detect protease [154]. Different from the direct fluorescence recovery induced by targets, the protease sensing was mediated by the histone triggered fluorescence recovery (Figure 10B). The histone could competitively bind to BPNSs through the electrostatic interaction, which released the donor from BPNSs and then led to the recovery of fluorescence. However, upon the addition of protease, the histone was dissociated into mini fragments and lost its affinity to BPNSs. As a result, the donor was reattached to BPNSs, and the fluorescence was quenched.

MXenes are regarded as a competent candidate in the development of FRET biosensing platforms owing to their intrinsic fluorescence-quenching ability and oxygen or hydroxyl terminated active surface. Zhang et al. [37] proposed a $\mathrm{Ti}_{3} \mathrm{C}_{2}$ MXene-based FRET sensor for quantitative detection of exosomes. The sensor was designed by using the $\mathrm{Cy}_{3}$-labeled $\mathrm{CD} 63$ aptamer/Ti ${ }_{3} \mathrm{C}_{2}$ as the FRET pair. The fluorescence of $\mathrm{Cy} 3$ greatly recovered because of the binding of aptamer to CD63 protein that was abundant on the exosomes surface. The LOD of exosomes was found to be $1.4 \times 10^{3}$ particles $/ \mathrm{ml}$. The authors emphasized that the developed sensor exhibited much better sensitivity than other reported studies and conventional enzyme-linked immunosorbent assay method.

As a summary, we mainly highlight the utilization of TMDs, $\mathrm{MnO}_{2}$, BP, 2D-MOFs, and MXenes as the nanoquencher for the development of FRET sensors. Typically, the probe molecules are first adsorbed on the surface of the $2 \mathrm{D}$ nanomaterials, resulting in the quenching of fluorescence of probe molecules. Then the target molecules interact with the probe molecules, leading to the release of probe molecules from the $2 \mathrm{D}$ nanomaterials and the recovery of fluorescence. There is one exception that most $\mathrm{MnO}_{2}$-based FRET sensors are relying on the reduction of $\mathrm{MnO}_{2}$ nanosheets by the targets. Owing to the broadspectrum quenching capability of these $2 \mathrm{D}$ nanomaterials, the emission wavelength of the donors could locate from the near-ultraviolet region to the NIR region. Moreover, the quenching efficiency of 2D nanomaterials is better than traditional nanoquenchers for their long range of quenching effect.

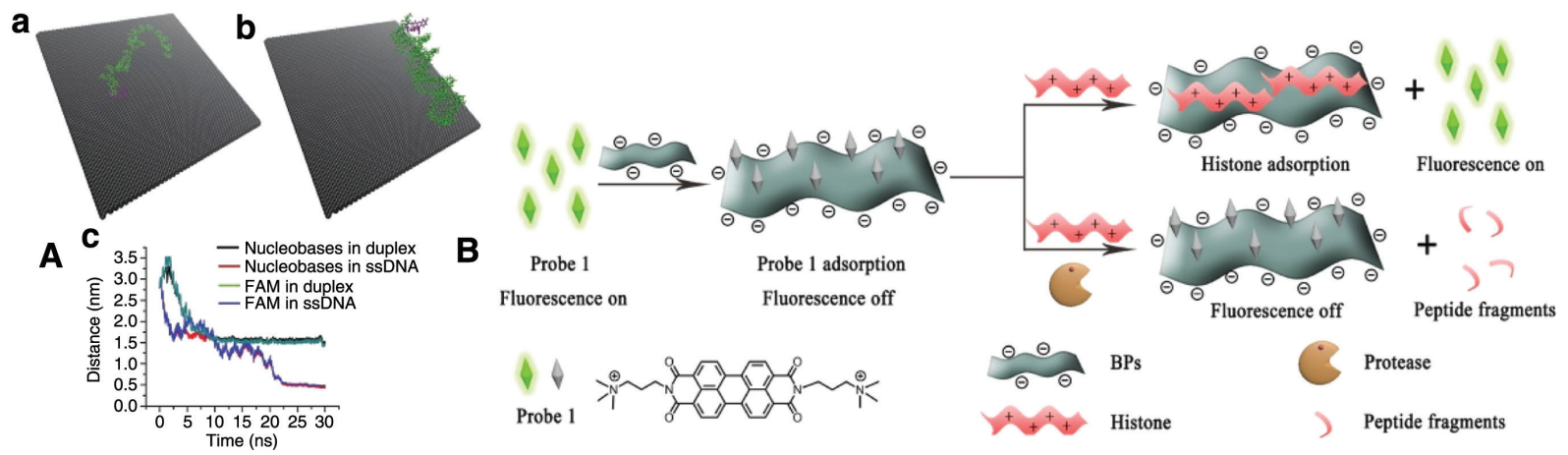

Figure 10: FRET sensors using BP nanosheets as the fluorescence quencher.

(A) Final snapshots of the MD simulation of ssDNA (a) and DNA/RNA duplex (b) mixing with the monolayer BP. (c) The real-time average distance between the nucleobases and the BP plane, the FAM and the BP plane under different circumstances. Reprinted with permission from Zhou et al. [69]. Copyright (2018) The Royal Society of Chemistry. (B) Schematic representation of a protease assay based on BP nanosheets. Reprinted with permission from Hu et al. [154]. Copyright (2018) Elsevier. 


\section{Conclusion}

In this review, we have discussed the recent advances in the application of 2D nanomaterials toward FRET sensing. Two-dimensional nanomaterials have been extensively studied and used as donors and acceptors in the development of FRET sensors due to their inherent advantages over traditional nanomaterials such as large specific surface area, high thermal and chemical stability, good optical properties, and biocompatibility. Nevertheless, the study on 2D nanomaterials for FRET sensing application is still at early stage, and many challenges still exist to be overcome. Herein, we present the difficulties and challenges that this field is facing, and based on this, we propose several research directions for the 2D nanomaterial-based FRET sensing applications.

In the first place, although the synthetic technology of 2D nanomaterials is growing rapidly, it is still difficult to obtain high-quality and highly uniform 2D nanomaterials, which is not beneficial to construct FRET sensors with good repeatability. Sonication exfoliation is the most common preparation method for 2D nanomaterials used in the FRET sensors. The main advantage of sonication exfoliation is its relatively low cost, simple process, high production yield, and high dispersity. However, the 2D nanomaterials exfoliated by this method are not satisfied in the size and thickness uniformity. Controlled centrifugation is a promising method to obtain dispersions of 2D nanomaterials with different size distributions [155, 156]. As the quality and size of 2D nanomaterials strongly depend on the sonication time, solvent, additive, ultrasonic power, and temperature during the sonication procedure, figuring out the underlying relationship would definitely benefit to the controllable preparation of highquality 2D nanomaterials [157].

Second, the fluorescent 2D nanomaterials are restricted by low quantum yield and broad emission band in comparison with semiconductive QDs, which would affect the efficiency of energy transfer [158]. Typically, there are four important factors that determine the quantum yield of 2D nanomaterials: molecule structure, size, surface structure, and external environment. Many efforts have been done to improve the quantum yield of 2D nanomaterials through modulating the above four factors. Taking graphene QDs, the most widely studied fluorescent 2D nanomaterial, as an example, various methods have been proposed by the researchers to enhance the quantum yield including size modification [159, 160], chemical doping [161-165], surface functionalization [166], and changing the solvent [167]. The quantum yield of graphene QDs could be increased to as high as $74.5 \%$ by codoping with nitrogen and sulfur [164]. However, the methods to improve the quantum yield of other 2D-QDs are rarely studied. Even the origin of PL of most 2D-QDs is not completely understood [168]. Taking TMD QDs as an example, the quantum yield of most reported TMD QDs was less than $5 \%$. Only in one study, the $\mathrm{MoS}_{2}$ QDs, which were synthetized through normal hydrothermal method, exhibited a high quantum yield of $13.4 \%$ [55]. Thus, the PL properties of 2D-QDs should be further explored, and more work on strategies for precise regulation of the PL behavior of 2D-QDs should be done.

Last but not the least, although 2D nanomaterials are known to possess abundant active sites for molecules binding, they also have the limitation of low specificity, which is not beneficial for the selective sensing. The issue is severe for FRET sensing as many targets are detected merely based on the overlapping of their absorption spectrum and the emission spectrum of donors rather than specific recognition. Surface modification or functionalization is a promising way to solve this problem as the accompanying rich chemical groups could provide more opportunities for enhancing the selectivity of 2D nanomaterials toward sensing elements. In addition, the elaborate design of the FRET sensors, including the choice of $2 \mathrm{D}$ nanomaterials, probe molecules, and experimental conditions, could greatly improve the selectivity of FRET sensors. For instance, with the assistance of first-principles calculations, the interactions of DNA with 2D nanomaterials could be predicted, which helps the researchers to choose appropriate 2D nanomaterial for the detection of target analysts [23].

\section{Abbreviations}

$\begin{array}{ll}\text { 2D } & \text { Two-dimensional } \\ \text { 6-MP } & \text { 6-Mercaptopurine } \\ \text { 6-TG } & \text { 6-Thioguanine } \\ \text { AA } & \text { Ascorbic acid } \\ \text { AuNCs } & \text { Gold nanoclusters } \\ \text { AuNPs } & \text { Gold nanoparticles } \\ \text { AuNRs } & \text { Gold nanorods } \\ \text { BP } & \text { Black phosphorene } \\ \text { BPNSs } & \text { BP nanosheets } \\ \text { BSA } & \text { Bovine serum albumin } \\ \text { CNTs } & \text { Carbon nanotubes } \\ \text { Cys } & \text { Cystamine } \\ \text { DAP } & \text { 2,3-Diaminophenazine } \\ \text { DMF } & \text { Dimethylfumarate } \\ \text { DMSO } & \text { Dimethylsulfoxide } \\ \text { DSN } & \text { Duplex-specific nuclease } \\ \text { EP } & \text { Epinephrine } \\ \text { g-C } \mathrm{N}_{4} & \text { Graphitic-phase } \mathrm{C}_{3} \mathrm{~N}_{4} \\ \text { GQDs } & \text { Graphene quantum dots }\end{array}$




$\begin{array}{ll}\text { GSH } & \text { Glutathione } \\ \text { HCRs } & \text { Hybridization chain reactions } \\ \text { IBV } & \text { Infectious bronchitis virus } \\ \text { LOD } & \text { Limit of detection } \\ \text { MBs } & \text { Molecular beacons } \\ \text { MD } & \text { Molecular dynamics } \\ \text { MES } & \text { 2-(N-morpholino)ethanesulfonic acid } \\ \text { MOFs } & \text { Metal-organic frameworks } \\ \text { NFZ } & \text { Nitrofurazone } \\ \text { NMM } & \text { N-methylmaleimide } \\ \text { NMP } & \text { N-methyl pyrrolidone } \\ \text { NPs } & \text { Nanoparticles } \\ \text { NRs } & \text { Nanorods } \\ \text { NW } & \text { Nanowire } \\ \text { PBA } & \text { 4-Mercaptophenylboronic acid } \\ \text { PCRs } & \text { Polymerase chain reactions } \\ \text { PL } & \text { Photoluminescence } \\ \text { PLL } & \text { Poly(L-lysine) } \\ \text { PLNPs } & \text { Persistent luminescence nanoparticles } \\ \text { R6G } & \text { Rhodamine 6G } \\ \text { FRET } & \text { Fluorescence resonant energy transfer } \\ \text { RBED } & \text { Rhodamine B ethylenediamine } \\ \text { rGO } & \text { Reduced graphene oxide } \\ \text { RhB } & \text { Rhodamine B } \\ \text { UCNPs } & \text { Upconversion nanoparticles } \\ \text { TMDs } & \text { Transition metal dichalcogenides } \\ \text { VdW } & \text { Van der Waals }\end{array}$

Acknowledgments: This work was supported by the Project from National Natural Science Foundation of China (61775148, 61527827, Funder Id: http://dx.doi. org/10.13039/501100001809), National Key Research and Development Program of China (2017YFB0403804), Guangdong Natural Science Foundation and Province Project (2017B020210006, 2018A030310544), and Shenzhen Science and Technology R\&D and Innovation Foundation (JCYJ20180305124754860).

\section{References}

[1] Förster T. Energiewanderung Und Fluoreszenz. Naturwissenschaften 1946;33:166-75.

[2] Zhang Y, Lim C-K, Dai Z, et al. Photonics and optoelectronics using nano-structured hybrid perovskite media and their optical cavities. Phys Rep 2019;795:1-51.

[3] Jares-Erijman EA, Jovin TM. FRET imaging. Nat Biotechnol 2003;21:1387-95.

[4] Jin B, Wang S, Lin M, et al. Upconversion nanoparticles based FRET aptasensor for rapid and ultrasenstive bacteria detection. Biosens Bioelectron 2017;90:525-33.

[5] Liu L, Zhang H, Song D, Wang Z. An upconversion nanoparticlebased fluorescence resonance energy transfer system for effectively sensing caspase-3 activity. Analyst 2018;143:761-7.

[6] Ma Y, Zhang H, Liu F, Wu Z, Mao H. Highly sensitive detection of DNA methylation level by using quantum dots-based FRET method. Nanoscale 2015;7:17547.
[7] Wu L, Guo Q, Liu Y, Sun Q. FRET-based ratiometric fluorescent probe for detection of $\mathrm{Zn}^{2+}$ using a dual-emission silica-coated quantum dots mixture. Anal Chem 2015;87:5318.

[8] Qin L, He X, Chen L, Zhang Y. Turn-on fluorescent sensing of glutathione S-transferase at near-infrared region based on FRET between gold nanoclusters and gold nanorods. ACS Appl Mater Inter 2015;7:5965-71.

[9] Lin Z, Zhang G, Yang W, Qiu B, Chen G. CEA fluorescence biosensor based on the FRET between polymer dots and $\mathrm{Au}$ nanoparticles. Chem Commun 2012;48:9918-20.

[10] Nasirian V, Chabok A, Barati A, Rafienia M, Arabi MS, Shamsipur M. Ultrasensitive aflatoxin B1 assay based on FRET from aptamer labelled fluorescent polymer dots to silver nanoparticles labeled with complementary DNA. Microchim Acta 2017;184:4655-62.

[11] Cheng X, Cen Y, Xu G, et al. Aptamer based fluorometric determination of ATP by exploiting the FRET between carbon dots and graphene oxide. Microchim Acta 2018;185:144.

[12] Qaddare SH, Salimi A. Amplified fluorescent sensing of DNA using luminescent carbon dots and AuNPs/GO as a sensing Platform: A novel coupling of FRET and DNA hybridization for homogeneous HIV-1 gene detection at femtomolar level. Biosens Bioelectron 2017;89:773-80.

[13] Shi J, Chan C, Pang Y, et al. A fluorescence resonance energy transfer (FRET) biosensor based on graphene quantum dots (GQDs) and gold nanoparticles (AuNPs) for the detection of mecA gene sequence of Staphylococcus aureus. Biosens Bioelectron 2015;67:595-600.

[14] Yu M, Wang H, Fu F, Li L, Song E. A Dual recognition FRET-based platform for one-step sensitive detection of pathogenic bacteria using fluorescent vancomycin-gold nanoclusters and aptamer-gold nanoparticles. Anal Chem 2017;89:4085-90.

[15] Zhou J, Cao Z, Panwar N, et al. Functionalized gold nanorods for nanomedicine: past, present and future. COORDIN CHEM REV 2017;352:15-66.

[16] Tian J, Zhao H, Liu M, Chen Y, Quan X. Detection of influenza $A$ virus based on fluorescence resonance energy transfer from quantum dots to carbon nanotubes. Anal Chim Acta 2012;723:83-7.

[17] Qian ZS, Shan XY, Chai LJ, Ma JJ, Chen JR, Feng H. DNA nanosensor based on biocompatible graphene quantum dots and carbon nanotubes. Biosens Bioelectron 2014;60:64-70.

[18] Liu J, Jiang X, Zhang R, et al. MXene-enabled electrochemical microfluidic biosensor: applications toward multicomponent continuous monitoring in whole blood. Adv Opt Mater 2019;29:1807326.

[19] Tao W, Kong N, Ji X, et al. Emerging two-dimensional monoelemental materials (Xenes) for biomedical applications. Chem Soc Rev 2019;48:2891-912.

[20] Qiu M, Wang D, Liang W, et al. Novel concept of the smart NIR-light-controlled drug release of black phosphorus nanostructure for cancer therapy. Proc Natl Acad Sci USA 2018;115:501-6.

[21] Qiu M, Ren WX, Jeong T, et al. Omnipotent phosphorene: a next-generation, two-dimensional nanoplatform for multidisciplinary biomedical applications. Chem Soc Rev 2018;47:5588-601.

[22] Qiu M, Singh A, Wang D, et al. Biocompatible and biodegradable inorganic nanostructures for nanomedicine: silicon and black phosphorus. Nano Today 2019;25:135-55. 
[23] Xue T, Liang W, Li Y, et al. Ultrasensitive detection of miRNA with an antimonene-based surface plasmon resonance sensor. Nat Commun 2019;10:28.

[24] Xie Z, Chen S, Duo Y, et al. Biocompatible two-dimensional titanium nanosheets for multimodal imaging-guided cancer theranostics. ACS Appl Mater Inter 2019;11:22129-40.

[25] Xie Z, Wang D, Fan T, et al. Black phosphorus analogue tin sulfide nanosheets: synthesis and application as near-infrared photothermal agents and drug delivery platforms for cancer therapy. J Mater Chem B 2018;6:4747-55.

[26] Zhang X-L, Zheng C, Guo S-S, Li J, Yang H-H, Chen G. Turn-on fluorescence sensor for intracellular imaging of glutathione using g- $\mathrm{C}_{3} \mathrm{~N}_{4}$ nanosheet- $\mathrm{MnO}_{2}$ sandwich nanocomposite. Anal Chem 2014;86:3426-34.

[27] Jiang X, Zhang L, Liu S, et al. Ultrathin metal-organic framework: an emerging broadband nonlinear optical material for ultrafast photonics. Adv Opt Mater 2018;6:1800561.

[28] Xing C, Xie Z, Liang Z, et al. 2D nonlayered selenium nanosheets: facile synthesis, photoluminescence, and ultrafast photonics. Adv Opt Mater 2017;5:1700884.

[29] Huang Y, Yan F, Xu J, et al. The FRET performance and aggregation-induced emission of two-dimensional organic-inorganic perovskite, and its application to the determination of $\mathrm{Hg}$ (II). Microchim Acta 2017;184:3513-9.

[30] Barua S, Dutta HS, Gogoi S, Devi R, Khan R. Nanostructured $\mathrm{MoS}_{2}$-based advanced biosensors: a review. ACS Appl Nano Mater 2018;1:2-25.

[31] Galande C, Mohite AD, Naumov AV, et al. Quasi-molecular fluorescence from graphene oxide. Sci Rep 2011;1:85.

[32] Swathi RS, Sebastian KL. Resonance energy transfer from a dye molecule to graphene. J Chem Phys 2008;129:719.

[33] Swathi RS, Sebastian KL. Long range resonance energy transfer from a dye molecule to graphene has (distance)(-4) dependence. J Chem Phys 2009;130:086101.

[34] Yan L, Shi H, Sui X, Deng Z, Gao L. MoS 2 -DNA and $\mathrm{MoS}_{2}$ based sensors. RSC Adv 2017;7:23573-82.

[35] Zhou J, Li Z, Ying M, et al. Black phosphorus nanosheets for rapid microRNA detection. Nanoscale 2018;10:5060-6.

[36] Huang W, Li C, Gao L, et al. Emerging black phosphorus analogue nanomaterials for high-performance device applications. J Mater Chem C 2020;8:1172-97.

[37] Zhang Q, Wang F, Zhang H, Zhang Y, Liu M, Liu Y. Universal $\mathrm{Ti}_{3} \mathrm{C}_{2}$ MXenes based self-standard ratiometric fluorescence resonance energy transfer platform for highly sensitive detection of exosomes. Anal Chem 2018;90:12737-44.

[38] Deng R, Xie X, Vendrell M, Chang Y-T, Liu X. Intracellular glutathione detection using $\mathrm{MnO}_{2}$-nanosheet-modified upconversion nanoparticles. J Am Chem Soc 2011;133:20168-71.

[39] Zheng P, Wu N. Fluorescence and sensing applications of graphene oxide and graphene quantum dots: a review. Chem Asian J 2017;12:2343-53.

[40] Tian F, Jing L, Shi J, Yang M. Graphene and graphene-like two-denominational materials based fluorescence resonance energy transfer (FRET) assays for biological applications. Biosens Bioelectron 2017;89:123-35.

[41] Tong L, Wei H, Zhang S, Xu H. Recent advances in plasmonic sensors. Sensors 2014;14:7959-73.

[42] Ge Y, Zhu Z, Xu Y, et al. Broadband nonlinear photoresponse of $2 \mathrm{D} \mathrm{TiS}_{2}$ for ultrashort pulse generation and all-optical thresholding devices. Adv Opt Mater 2018;6:1701166.
[43] Jiang Y, Miao L, Jiang G, et al. Broadband and enhanced nonlinear optical response of $\mathrm{MoS}_{2}$ /graphene nanocomposites for ultrafast photonics applications. Sci Rep 2015;5:16372.

[44] Zhang H, Lu S, Zheng J, et al. Molybdenum disulfide $\left(\mathrm{MoS}_{2}\right)$ as a broadband saturable absorber for ultra-fast photonics. Opt Express 2014;22:7249-60.

[45] Han W, Zang C, Huang Z, et al. Enhanced photocatalytic activities of three-dimensional graphene-based aerogel embedding $\mathrm{TiO}_{2}$ nanoparticles and loading $\mathrm{MoS}_{2}$ nanosheets as Co-catalyst. Int J Hydrogen Energ 2014;39:19502-12.

[46] Arul NS, Nithya VD. Molybdenum disulfide quantum dots: synthesis and applications. RSC Adv 2016;6:65670-82.

[47] Shanmugaraj K, John SA. Water-soluble $\mathrm{MoS}_{2}$ quantum dots as effective fluorescence probe for the determination of bilirubin in human fluids. Spectrochim Acta A 2019;215:290-6.

[48] Yu X, Hu L, Zhang F, Wang M, Xia Z, Wei W. MoS 2 quantum dots modified with a labeled molecular beacon as a ratiometric fluorescent gene probe for FRET based detection and imaging of microRNA. Microchim Acta 2018;185.

[49] Zhang F, Wang M, Zeng D, Zhang H, Li Y, Su X. A molybdenum disulfide quantum dots-based ratiometric fluorescence strategy for sensitive detection of epinephrine and ascorbic acid. Anal Chim Acta 2019;1089:123-30.

[50] Zhang F, Liu H, Liu Q, Su X. An enzymatic ratiometric fluorescence assay for 6-mercaptopurine by using $\mathrm{MoS}_{2}$ quantum dots. Microchim Acta 2018;185:540.

[51] Swaminathan H, Ramar V, Balasubramanian K. Excited-state electron and energy transfer dynamics between $2 \mathrm{D} \mathrm{MoS}_{2}$ and GO/RGO for turn ON BSA/HSA sensing. J Phys Chem C 2017;121:12585-92.

[52] Chen J, Li Y, Huang Y, Zhang H, Chen X, Qiu H. Fluorometric dopamine assay based on an energy transfer system composed of aptamer-functionalized $\mathrm{MoS}_{2}$ quantum dots and $\mathrm{MoS}_{2}$ nanosheets. Microchim Acta 2019;186:58.

[53] Swaminathan H, Balasubramanian K. Förster resonance energy transfer between $\mathrm{MoS}_{2}$ quantum dots and polyaniline for turn-on bovine serum albumin sensing. Sens Actuator B Chem 2018;264:337-43.

[54] Balasubramanian K, Swaminathan H. Highly sensitive sensing of glutathione based on Förster resonance energy transfer between $\mathrm{MoS}_{2}$ donors and rhodamine $6 \mathrm{G}$ acceptors and its insight. Sens Actuator B Chem 2018;259:980-9.

[55] Li W, Shi Y, Hu X, et al. Visual detection of nitrite in sausage based on a ratiometric fluorescent system. Food Control 2019;106:106704.

[56] Guo X, Wang Y, Wu F, Ni Y, Kokot S. The use of tungsten disulfide dots as highly selective, fluorescent probes for analysis of nitrofurazone. Talanta 2015;144:1036-43.

[57] Ying TY, Sofer Z, Mayorgamartinez CC, Pumera M. Black phosphorus nanoparticles as a novel fluorescent sensing platform for nucleic acid detection. Mater Chem Front 2017;1:1130-6.

[58] Zhou Y-J, Li L, Wan Y-H, Chen T-T, Chu X. 2D g- $\mathrm{C}_{3} \mathrm{~N}_{4}-\mathrm{MnO}_{2}$ nanocomposite for sensitive and rapid turn-on fluorescence detection of $\mathrm{H} 2 \mathrm{O} 2$ and glucose. Anal Methods 2018;10: 5084-90.

[59] Men C, Li CH, Wei XM, et al. A sensitive and low background fluorescent sensing strategy based on $\mathrm{g}-\mathrm{C}_{3} \mathrm{~N}_{4}-\mathrm{MnO}_{2}$ sandwich nanocomposite and liposome amplification for ricin detection. Analyst 2018;143:5764-70. 
[60] Han J, Zou HY, Gao MX, Huang CZ. A graphitic carbon nitride based fluorescence resonance energy transfer detection of riboflavin. Talanta 2016;148:279-84.

[61] Hatamie A, Marahel F, Sharifat A. Green synthesis of graphitic carbon nitride nanosheet $\left(\mathrm{g}-\mathrm{C}_{3} \mathrm{~N}_{4}\right)$ and using it as a label-free fluorosensor for detection of metronidazole via quenching of the fluorescence. Talanta 2018;176:518-25.

[62] Guo X, Wu F, Ni Y, Kokot S. Synthesizing a nano-composite of BSA-capped Au nanoclusters/graphitic carbon nitride nanosheets as a new fluorescent probe for dopamine detection. Anal Chim Acta 2016;942:112-20.

[63] Ha HD, Dong JH, Choi JS, Park M, Seo TS. Dual role of blue luminescent $\mathrm{MoS}_{2}$ quantum dots in fluorescence resonance energy transfer phenomenon. Small 2014;10:3858-62.

[64] Zhang M, Wu Q, Zhang F, et al. 2D Black phosphorus saturable absorbers for ultrafast photonics. Adv Opt Mater 2019;7:1970001.

[65] Zhou Y, Zhang M, Guo Z, et al. Recent advances in black phosphorus-based photonics, electronics, sensors and energy devices. Mater Horiz 2017;4:997-1019.

[66] Zhang X, Xie HM, Liu ZD, et al. Black phosphorus quantum dots. Angew Chem Int Edit 2015;54:3653-7.

[67] Gu W, Pei X, Cheng Y, et al. Black phosphorus quantum dots as the ratiometric fluorescence probe for trace mercury ion detection based on inner filter effect. ACS Sens 2017;2:576-82.

[68] Gu W, Yan Y, Pei X, Zhang C, Ding C, Xian Y. Fluorescent black phosphorus quantum dots as label-free sensing probes for evaluation of acetylcholinesterase activity. Sensor Actuat B-Chem 2017;250:601-7.

[69] Zhou J, Li Z, Ying M, Liu M, Xu G. Black phosphorus nanosheets for rapid microRNA detection. Nanoscale 2018;10:5060-4.

[70] Li P, Yao C, Yang T, Wang Z, Bao Q. Two-dimensional $\mathrm{CH}_{3} \mathrm{NH}_{3} \mathrm{Pbl}_{3}$ perovskite nanosheets for ultrafast pulsed fiber lasers. ACS Appl Mater Inter 2017;9:12759.

[71] Xiang Q, Yupeng Z, Qingdong O, et al. Photonics and optoelectronics of 2D metal-halide perovskites. Small 2018;14:1800682.

[72] Niu Y, Feng Z, Bai Z, Dong Y, Zhong H. Aggregation-induced emission features of organometal halide perovskites and their fluorescence probe applications. Adv Opt Mater 2014;3:112-9.

[73] Zhu C, Zeng Z, Li H, Li F, Fan C, Zhang H. Single-layer $\mathrm{MoS}_{2}$ based nanoprobes for homogeneous detection of biomolecules. J Am Chem Soc 2013;135:5998.

[74] Huang J, Ye L, Gao X, Li H, Xu J, Li Z. Molybdenum disulfidebased amplified fluorescence DNA detection using hybridization chain reactions. J Mater Chem B 2015;3:2395-401.

[75] Huang Y, Shi Y, Yang HY, Ai Y. A novel single-layered MoS nanosheet based microfluidic biosensor for ultrasensitive detection of DNA. Nanoscale 2015;7:2245-9.

[76] Ying Z, Bing Z, Changfeng Z, et al. Single-layer transition metal dichalcogenide nanosheet-based nanosensors for rapid, sensitive, and multiplexed detection of DNA. Adv Mater 2015;27:935-9.

[77] Loo AH, Bonanni A, Pumera M. Strong dependence of fluorescence quenching on the transition metal in layered transition metal dichalcogenide nanoflakes for nucleic acid detection. Analyst 2016;141:4654-8.

[78] Liu Y-F, Xue J-T, Yan H-J, Yang L-J, Liu W, Sun X-D. A Dual- signal amplification method for DNA detection based on exonuclease III and fluorescence quenching ability of $\mathrm{MoS}_{2}$ nanosheet. Chinese I Anal Chem 2017;45:303-7.
[79] Oudeng G, Au M, Shi J, Wen C, Yang M. One-step in situ detection of miRNA-21 expression in single cancer cells based on biofunctionalized $\mathrm{MoS}_{2}$ nanosheets. ACS Appl Mater Inter 2018;10:350-60.

[80] Xiao M, Chandrasekaran AR, Ji W, et al. Affinity-modulated molecular beacons on $\mathrm{MoS}_{2}$ nanosheets for microRNA detection. ACS Appl Mater Inter 2018;10:35794-800.

[81] Xiao M, Man T, Zhu C, et al. $\mathrm{MoS}_{2}$ Nanoprobe for microRNA quantification based on duplex-specific nuclease signal amplification. ACS Appl Mater Inter 2018;10:7852-8.

[82] Bian F, Sun L, Cai L, et al. Molybdenum disulfide-integrated photonic barcodes for tumor markers screening. Biosens Bioelectron 2019;133:199-204.

[83] Wang L, Dong L, Liu G, et al. Fluorometric determination of HIV DNA using molybdenum disulfide nanosheets and exonuclease III-assisted amplification. Microchim Acta 2019;186:286.

[84] Lan L, Chen D, Yao Y, et al. Phase-dependent fluorescence quenching efficiency of $\mathrm{MoS}_{2}$ nanosheets and their applications in multiplex target biosensing. ACS Appl Mater Inter 2018;10:42009-17.

[85] Wang L, Huang Z, Wang R, et al. Transition metal dichalcogenide nanosheets for visual monitoring PCR rivaling a real-time PCR instrument. ACS Appl Mater Inter 2018;10:4409-18.

[86] Ge J, Ou E-C, Yu R-Q, Chu X. A novel aptameric nanobiosensor based on the self-assembled DNA-MoS ${ }_{2}$ nanosheet architecture for biomolecule detection. J Mater Chem B 2014;2:625-8.

[87] Kong R-M, Ding L, Wang Z, You J, Qu F. A novel aptamer-functionalized $\mathrm{MoS}_{2}$ nanosheet fluorescent biosensor for sensitive detection of prostate specific antigen. Anal Bioanal Chem 2015;407:369-77.

[88] Kenry, Geldert A, Xiao Z, Hua Z, Lim CT. Highly sensitive and selective aptamer-based fluorescence detection of a malarial biomarker using single-layer $\mathrm{MoS}_{2}$ nanosheets. ACS Sens 2016;1:1315-21.

[89] Dhenadhayalan N, Yadav K, Sriram MI, Lee H-L, Lin K-C. Ultrasensitive DNA sensing of a prostate-specific antigen based on 2D nanosheets in live cells. Nanoscale 2017;9:12087-95.

[90] Geldert A, Kenry, Lim CT. Paper-based $\mathrm{MoS}_{2}$ nanosheetmediated FRET aptasensor for rapid malaria diagnosis. Sci Rep 2017;7:17510.

[91] Geldert A, Kenry, Zhang X, Zhang H, Lim CT. Enhancing the sensing specificity of a $\mathrm{MoS}_{2}$ nanosheet-based FRET aptasensor using a surface blocking strategy. Analyst 2017;142:2570-7.

[92] Shi J, Lyu J, Tian F, Yang M. A fluorescence turn-on biosensor based on graphene quantum dots (GQDs) and molybdenum disulfide $\left(\mathrm{MoS}_{2}\right)$ nanosheets for epithelial cell adhesion molecule (EpCAM) detection. Biosens Bioelectron 2017;93:182-8.

[93] Xu S, Feng X, Gao T, et al. Aptamer induced multicoloured Au $\mathrm{NCs}-\mathrm{MoS}_{2}$ "switch on" fluorescence resonance energy transfer biosensor for dual color simultaneous detection of multiple tumor markers by single wavelength excitation. Anal Chim Acta 2017;983:173-80.

[94] Yang H, Jie X, Wang L, Zhang Y, Wang M, Wei W. An array consisting of glycosylated quantum dots conjugated to $\mathrm{MoS}_{2}$ nanosheets for fluorometric identification and quantitation of lectins and bacteria. Microchim Acta 2018;185:512.

[95] Zhao L, Cheng M, Liu G, et al. A fluorescent biosensor based on molybdenum disulfide nanosheets and protein aptamer for sensitive detection of carcinoembryonic antigen. Sens Actuator B Chem 2018;273:185-90. 
[96] Mao K, Wu Z, Chen Y, Zhou X, Shen A, Hu J. A novel biosensor based on single-layer $\mathrm{MoS}_{2}$ nanosheets for detection of $\mathrm{Ag}^{+}$. Talanta 2015;132:658-63.

[97] Srinivasan K, Subramanian K, Murugan K, Dinakaran K. Sensitive fluorescence detection of mercury(II) in aqueous solution by the fluorescence quenching effect of $\mathrm{MoS}_{2}$ with DNA functionalized carbon dots. Analyst 2016;141:6344-52.

[98] Jia L, Ding L, Tian J, et al. Aptamer loaded $\mathrm{MoS}_{2}$ nanoplates as nanoprobes for detection of intracellular ATP and controllable photodynamic therapy. Nanoscale 2015;7:15953-61.

[99] Qu F, Liu Y, Kong R, You J. A versatile DNA detection scheme based on the quenching of fluorescent silver nanoclusters by $\mathrm{MoS}_{2}$ nanosheets: application to aptamer-based determination of hepatitis B virus and of dopamine. Microchim Acta 2017;184:4417-24.

[100] Wang Y, Ma T, Ma S, et al. Fluorometric determination of the antibiotic kanamycin by aptamer-induced FRET quenching and recovery between $\mathrm{MOS}_{2}$ nanosheets and carbon dots. Microchim Acta 2017;184:203-10.

[101] Chen K, Zhang W, Zhang Y, et al. Label-free fluorescence aptasensor for sensitive determination ofbisphenol $\mathrm{S}$ by the salt-adjusted FRET between CQDs and $\mathrm{MoS}_{2}$. Sens Actuator B Chem 2018;259:717-24.

[102] Fan YY, Mou ZL, Wang M, et al. Chimeric aptamers-based and $\mathrm{MoS}_{2}$ nanosheet-enhanced label-free fluorescence polarization strategy for adenosine triphosphate detection. Anal Chem 2018;90:13708.

[103] Khan IM, Zhao S, Niazi S, et al. Silver nanoclusters based FRET aptasensor for sensitive and selective fluorescent detection of T-2 toxin. Sens Actuator B Chem 2018;277:328-35.

[104] Liu X, Chen H, Lin J, Li Y, Guo L. Exfoliation of transition-metal dichalcogenides using ATP in aqueous solution. Chem Commun 2019;55:2972-5.

[105] Deng H, Yang X, Gao Z. MoS 2 nanosheets as an effective fluorescence quencher for DNA methyltransferase activity detection. Analyst 2015;140:3210-5.

[106] Singh P, Gupta R, Sinha M, Kumar R, Bhalla V. $\mathrm{MoS}_{2}$ based digital response platform for aptamer based fluorescent detection of pathogens. Microchim Acta 2016;183:1501-6.

[107] Xiang X, Shi J, Huang F, Zheng M, Deng Q, Xu J. MoS nanosheet-based fluorescent biosensor for protein detection via terminal protection of small-molecule-linked DNA and exonuclease III-aided DNA recycling amplification. Biosens Bioelectron 2015;74:227-32.

[108] Gogoi S, Khan R. Förster resonance energy transfer (FRET) between carbon dots $/ \mathrm{MoS}_{2}$ based fluorescence immunosensor for sensitive detection of cardiac troponin T. Phys Chem Chem Phys 2018;20:16501-9.

[109] Lv J, Zhao S, Wu S, Wang Z. Upconversion nanoparticles grafted molybdenum disulfide nanosheets platform for microcystin-LR sensing. Biosens Bioelectron 2017;90:203-9.

[110] Xuan W, Neethirajan S. Immunosensor based on antibodyfunctionalized $\mathrm{MoS}_{2}$ for rapid detection of avian coronavirus on cotton thread. IEEE Sens J 2018;18:4358-63.

[111] Xi Q, Zhou D-M, Kan Y-Y, et al. Highly sensitive and selective strategy for microRNA detection based on $\mathrm{WS}_{2}$ nanosheet mediated fluorescence quenching and duplex-specific nuclease signal amplification. Anal Chem 2014;86:1361-5.

[112] Yuan Y, Li R, Liu Z. Establishing water-soluble layered WS $_{2}$ nanosheet as a platform for biosensing. Anal Chem 2014;86:3610-5.
[113] Qin Y, Ma Y, Jin X, Zhang L, Ye G, Zhao S. A sensitive fluorescence turn-on assay of bleomycin and nuclease using $\mathrm{WS}_{2}$ nanosheet as an effective sensing platform. Anal Chim Acta 2015;866:84-9.

[114] Wang S, Zhang Y, Ning Y, Zhang G-J. A WS 2 nanosheet-based platform for fluorescent DNA detection via PNA-DNA hybridization. Analyst 2015;140:434-9.

[115] Li J, Zhao Q, Tang Y. Label-free fluorescence assay of S1 nuclease and hydroxyl radicals based on water-soluble conjugated polymers and $\mathrm{WS}_{2}$ nanosheets. Sensors 2016;16:865.

[116] Zuo X, Zhang H, Zhu Q, Wang W, Feng J, Chen X. A dual-color fluorescent biosensing platform based on $\mathrm{WS}_{2}$ nanosheet for detection of $\mathrm{Hg} 2+$ and $\mathrm{Ag}+$. Biosens Bioelectron 2016;85:464-70.

[117] Ge J, Xin G, Du Y-H, et al. Highly sensitive fluorescence detection of mercury (II) ions based on $\mathrm{WS}_{2}$ nanosheets and T7 exonuclease assisted cyclic enzymatic amplification. Sens Actuator B Chem 2017;249:189-94.

[118] Sun X, Fan J, Fu C, et al. WS 2 and $\mathrm{MoS}_{2}$ biosensing platforms using peptides as probe biomolecules. Sci Rep 2017;7:10290.

[119] Zuo X, Dai H, Zhang H, Liu J, Ma S, Chen X. A peptide-WS nanosheet based biosensing platform for determination of beta-secretase and screening of its inhibitors. Analyst 2018;143:4585-91.

[120] Niazi S, Khan IM, Yu Y, et al. A “turnon” aptasensor for simultaneous and time-resolved fluorometric determination of zearalenone, trichothecenes A and aflatoxin B-1 using $\mathrm{WS}_{2}$ as a quencher. Microchim Acta 2019;186:575.

[121] Li N, Diao W, Han Y, Pan W, Zhang T, Tang B. $\mathrm{MnO}_{2}$-modified persistent luminescence nanoparticles for detection and imaging of glutathione in living cells and in vivo. Chem Eur J 2014;20:16488-91.

[122] Meng H-M, Jin Z, Lv Y, et al. Activatable two-photon fluorescence nanoprobe for bioimaging of glutathione in living cells and tissues. Anal Chem 2014;86:12321-6.

[123] Cai Q-Y, Li J, Ge J, et al. A rapid fluorescence "switch-on" assay for glutathione detection by using carbon dots $-\mathrm{MnO}_{2}$ nanocomposites. Biosens Bioelectron 2015;72:31-6.

[124] Wang Y, Jiang K, Zhu J, Zhang L, Lin H. A FRET-based carbon dot$\mathrm{MnO}_{2}$ nanosheet architecture for glutathione sensing in human whole blood samples. Chem Commun 2015;51:12748-51.

[125] Liu Z, Cai X, Lin X, et al. Signal-on fluorescent sensor based on GQDs- $\mathrm{MnO}_{2}$ composite for glutathione. Anal Methods 2016;8:2366-74.

[126] Wang H-B, Chen Y, Li Y, Liu Y-M. A sensitive fluorescence sensor for glutathione detection based on $\mathrm{MnO}_{2}$ nanosheetscopper nanoclusters composites. RSC Adv 2016;6:79526-32.

[127] Wang X, Wang D, Guo Y, et al. Fluorescent glutathione probe based on $\mathrm{MnO}_{2}$-phenol formaldehyde resin nanocomposite. Biosens Bioelectron 2016;77:299-305.

[128] Yan X, Song Y, Zhu C, et al. Graphene quantum dot- $\mathrm{MnO}_{2}$ nanosheet based optical sensing platform: a sensitive fluorescence "turn off-on" nanosensor for glutathione detection and intracellular imaging. ACS Appl Mater Inter 2016;8:21990-6.

[129] Cai Q, Zhang L, Geng X, Ge J, Li Z. A self-assembly fluorescence sensing platform for glutathione detection based on eco-friendly quantum dots and $\mathrm{MnO}_{2}$ nanosheets. J Nanosci Nanotechnol 2018;18:1709-15.

[130] Meng H-M, Zhao D, Li N, Chang J. A graphene quantum dotbased multifunctional two-photon nanoprobe for the detec- 
tion and imaging of intracellular glutathione and enhanced photodynamic therapy. Analyst 2018;143:4967-73.

[131] Mi Y, Lei X, Han H, Liang J, Liu L. A sensitive label- free FRET probe for glutathione based on CdSe/ZnS quantum dots and $\mathrm{MnO}_{2}$ nanosheets. Anal Methods 2018;10:4170-7.

[132] Revuri V, Cherukula K, Nafiujjaman M, Cho KJ, Park I-K, Lee Y-K. White-light-emitting carbon nano-onions: a tunable multichannel fluorescent nanoprobe for glutathione-responsive bioimaging. ACS Appl Nano Mater 2018;1:662-74.

[133] Sheng J, Jiang X, Wang L, Yang M, Liu Y-N. Biomimetic mineralization guided one-pot preparation of gold clusters anchored two-dimensional $\mathrm{MnO}_{2}$ nanosheets for fluorometric/magnetic bimodal sensing. Anal Chem 2018;90:2926-32.

[134] Shi W, Song B, Shi W, et al. Bimodal phosphorescence magnetic resonance imaging nanoprobes for glutathione based on $\mathrm{MnO}_{2}$ nanosheet $\mathrm{Ru}(\mathrm{II})$ complex nanoarchitecture. ACS Appl Mater Inter 2018;10:27681-91.

[135] Song Z-L, Dai X, Li M, et al. Biodegradable nanoprobe based on $\mathrm{MnO}_{2}$ nanoflowers and graphene quantum dots for near infrared fluorescence imaging of glutathione in living cells. Microchim Acta 2018;185:485.

[136] Sun J, Liu F, Yu W, et al. Highly sensitive glutathione assay and intracellular imaging with functionalized semiconductor quantum dots. Nanoscale 2019;11:5014-20.

[137] Wang H-B, Chen Y, Li N, Liu Y-M. A fluorescent glucose bioassay based on the hydrogen peroxide-induced decomposition of a quencher system composed of $\mathrm{MnO}_{2}$ nanosheets and copper nanoclusters. Microchim Acta 2017;184:515-23.

[138] Xiao T, Sun J, Zhao J, Wang S, Liu G, Yang X. FRET effect between fluorescent polydopamine nanoparticles and $\mathrm{MnO}_{2}$ nanosheets and its application for sensitive sensing of alkaline phosphatase. ACS Appl Mater Inter 2018;10:6560-9.

[139] Na W, Li N, Su X. Enzymatic growth of single-layer $\mathrm{MnO}_{2}$ nanosheets in situ: application to detect alkaline phosphatase and ascorbic acid in the presence of sulfanilic acid functionalized graphene quantum dots. Sens Actuator B Chem 2018;274:172-9.

[140] Yan X, Song Y, Zhu C, et al. $\mathrm{MnO}_{2}$ Nanosheet-carbon dots sensing platform for sensitive detection of organophosphorus pesticides. Anal Chem 2018;90:2618-24.

[141] Zhang Y, Li Y, Zhang C, et al. Fluorescence turn-on detection of alkaline phosphatase activity based on controlled release of PEl-capped $\mathrm{Cu}$ nanoclusters from $\mathrm{MnO}_{2}$ nanosheets. Anal Bioanal Chem 2017;409:4771-8.

[142] Hu Y, Zhang L, Geng X, Ge J, Liu H, Li Z. A rapid and sensitive turn-on fluorescent probe for ascorbic acid detection based on carbon dots $-\mathrm{MnO}_{2}$ nanocomposites. Anal Methods 2017;9:5653-8.

[143] Hu C, Kong XJ, Yu RQ, Chen TT, Chu X. MnO nanosheet-based fluorescence sensing platform for sensitive detection of endonuclease. Anal Sci 2017;33:783-8.

[144] Zhang Y, Zhang C, Chen J, et al. A real-time fluorescence turn-on assay for acetylcholinesterase activity based on the controlled release of a perylene probe from $\mathrm{MnO}_{2}$ nanosheets. J Mater Chem C 2017;5:4691-4.

[145] Han Y, Ye Z, Wang F, et al. Single-particle enumeration-based ultrasensitive enzyme activity quantification with fluorescent polymer nanoparticles. Nanoscale 2019;11:14793-801.

[146] Yang Q, Wang X, Peng H, et al. Ratiometric fluorescence and colorimetry dual-mode assay based on manganese dioxide nanosheets for visual detection of alkaline phosphatase activity. Sens Actuator B Chem 2020;302:127176.

[147] Garg D, Mehta A, Mishra A, Basu S. A sensitive turn on fluorescent probe for detection of biothiols using $\mathrm{MnO}_{2} @$ carbon dots nanocomposites. Spectrochim Acta A 2018;192:411-9.

[148] Song W-J. Intracellular DNA and microRNA sensing based on metal-organic framework nanosheets with enzyme-free signal amplification. Talanta 2017;170:74-80.

[149] Wang H-S, Li J, Li J-Y, Wang K, Ding Y, Xia X-H. Lanthanidebased metal-organic framework nanosheets with unique fluorescence quenching properties for two-color intracellular adenosine imaging in living cells. NPG Asia Mater 2017;9:354.

[150] Wang H-S, Liu H-L, Wang K, et al. Insight into the unique fluorescence quenching property of metal-organic frameworks upon DNA binding. Anal Chem 2017;89:11366-71.

[151] Yang S-P, Zhao W, Hu P-P, et al. Lanthanum-based metalorganic frameworks for specific detection of sudan virus RNA conservative sequences down to single-base mismatch. Inorganic Chemistry 2017;56:14880-7.

[152] Hai XM, Li N, Wang K, Zhang ZQ, Zhang J, Dang FQ. A fluorescence aptasensor based on two-dimensional sheet metalorganic frameworks for monitoring adenosine triphosphate. Anal Chim Acta 2018;998:60-6.

[153] Yang Q, Zhou L, Wu Y-X, et al. A two dimensional metalorganic framework nanosheets-based fluorescence resonance energy transfer aptasensor with circular strand-replacement DNA polymerization target-triggered amplification strategy for homogenous detection of antibiotics. Anal Chim Acta 2018;1020:1-8.

[154] Hu Z, Li Y, Hussain E, et al. Black phosphorus nanosheets based sensitive protease detection and inhibitor screening. Talanta 2019;197:270-6.

[155] Hanlon D, Backes C, Doherty E, et al. Liquid exfoliation of solvent-stabilized few-layer black phosphorus for applications beyond electronics. Nat Commun 2015;6:8563.

[156] Khan U, O’Neill A, Porwal H, May P, Nawaz K, Coleman JN. Size selection of dispersed, exfoliated graphene flakes by controlled centrifugation. Carbon 2012;50:470-5.

[157] Tan C, Cao X, Wu X-J, et al. Recent advances in ultrathin twodimensional nanomaterials. Chem Rev 2017;117:6225-331.

[158] Shen J, Zhu Y, Yang X, et al. Graphene quantum dots: emergent nanolights for bioimaging, sensors, catalysis and photovoltaic devices. ChemInform 2012;48:3686-99.

[159] Zhu S, Zhang J, Qiao C, et al. Strongly green-photoluminescent graphene quantum dots for bioimaging applications. Chem Commun 2011;47:6858-60.

[160] Li Y, Hu Y, Zhao Y, et al. An electrochemical avenue to greenluminescent graphene quantum dots as potential electronacceptors for photovoltaics. Adv Mater 2011;23:776-80.

[161] Sun J, Yang S, Wang Z, et al. Ultra-high quantum yield of graphene quantum dots: aromatic-nitrogen doping and photoluminescence mechanism. Part Part Syst Char 2015;32:434-40.

[162] Qu D, Zheng M, Zhang L, et al. Formation mechanism and optimization of highly luminescent $\mathrm{N}$-doped graphene quantum dots. Sci Rep 2014;4:5294.

[163] Wu ZL, Gao MX, Wang TT, Wan XY, Zheng LL, Huang CZ. A general quantitative $\mathrm{pH}$ sensor developed with dicyandiamide $\mathrm{N}$-doped high quantum yield graphene quantum dots. Nanoscale 2014;6:3868-74. 
[164] Xia C, Hai X, Chen XW, Wang JH. Simultaneously fabrication of free and solidified N, S-doped graphene quantum dots via a facile solvent-free synthesis route for fluorescent detection. Talanta 2017;168:269.

[165] Kharangarh PR, Umapathy S, Singh G. Investigation of sulfur related defects in graphene quantum dots for tuning photoluminescence and high quantum yield. Appl Surf Sci 2018;449:363-70.

[166] Qian Z, Ma J, Shan X, et al. Surface functionalization of graphene quantum dots with small organic molecules from photoluminescence modulation to bioimaging applications: an experimental and theoretical investigation. RSC Advances 2013;3:14571-9.

[167] Zhu Y, Wang G, Jiang H, Chen L, Zhang X. One-step ultrasonic synthesis of graphene quantum dots with high quantum yield and their application in sensing alkaline phosphatase. Chem Commun 2015;51:948-51.

[168] Cao X, Ding C, Zhang C, et al. Transition metal dichalcogenide quantum dots: synthesis, photoluminescence and biological applications. J Mater Chem B 2018;6:8011-36. 